\title{
LAMONT RADIOCARBON MEASUREMENTS VIII
}

\author{
WALLACE S. BROECKER and EDWIN A. OLSON**
}

Lamont Geological Observatory, Columbia University, Palisades, New York

Like Lamont VI (Broecker and Olson, 1959), this list contains only results on samples of known age (most of which formed during the past ten years). The measurements were made largely in order to gain an understanding of the distribution of radiocarbon within the dynamic carbon reservoir. Again, the data are not reported primarily with the idea of drawing new conclusions but rather to bring together in one place information which is presently scattered throughout the literature or which otherwise might remain unpublished.

Although the system of data presentation is essentially the same as that adopted in our previous paper, a change has been made in the normalization formula:

previous relationship (Lamont VI)

$$
\Delta \mathrm{C}^{14}=\delta \mathrm{C}^{14}-2 \delta \mathrm{C}^{13}\left(1+\frac{\delta \mathrm{C}^{14}}{1000}\right)-50.0
$$

revised relationship (as suggested by K. C. Munnich and J. C. Vogel, personal communication)

$$
\begin{aligned}
& \Delta=\delta \mathrm{C}^{14}-2 \delta \mathrm{C}^{13}\left(1+\frac{\delta \mathrm{C}^{14}}{1000}\right)-50.0\left(1+\frac{\delta \mathrm{C}^{14}}{1000}\right) \\
& \text { or } \\
& \Delta=\delta \mathrm{C}^{14}-\left(2 \delta \mathrm{C}^{13}+50\right)\left(1+\frac{\delta \mathrm{C}^{14}}{1000}\right)
\end{aligned}
$$

The symbol $\Delta$ has been substituted for $\Delta \mathrm{C}^{14}$ in the revised formula in order to eliminate confusion between the two modes of normalization. The $\left(1+\frac{\delta \mathrm{C}^{14}}{1000}\right)$ term replaces unity as a multiplier of the constant term, 50.0, in order to yield $\Delta$ values which reflect the exact per-millage change in the fractionation-normalized $\mathrm{C}^{14}$ concentrations. That this was not precisely the case with the previous system can be most easily demonstrated by considering an example. Two plant samples identical in $\mathrm{C}^{13} / \mathrm{C}^{12}$ ratio $\left(\delta \mathrm{C}^{13}=-25.0\right)$ but differing by a factor of 1.200 in $\mathrm{C}^{14} / \mathrm{C}^{12}$ ratio $\left(\delta \mathrm{C}_{1}{ }^{14}=0\right.$ and $\left.\delta \mathrm{C}_{2}{ }^{14}=200\right)$ would have $\Delta \mathrm{C}^{14}$ values of 0 and 210 per mil respectively and $\Delta$ values of 0 and 200 per mil. Thus, the old scale $\left(\Delta \mathrm{C}^{14}\right)$ would unnecessarily introduce an additional 10 per mil difference, while the corrected scale $(\Delta)$ would give a difference consistent with the measured $\mathrm{C}^{14}$ activities.

The convenience provided by the $\Delta$ scale should more than compensate for any confusion resulting from the change. $\Delta \mathrm{C}^{14}$ results can be easily converted to $\Delta$ results by the following relationship:

$$
\Delta=\Delta \mathrm{C}^{14}-\frac{\delta \mathrm{C}^{14}}{20}
$$

* Lamont Geological Observatory Contribution no. 495.

** Present address: Division of Science, Whitworth College, Spokane, Washington. 
It can be seen that the scale difference exceeds 5 per mil only for samples having $\mathrm{C}^{14}$ concentrations differing from 0.95 oxalic acid by more than 100 per mil.

The scale change in no way alters the method of computing $\delta \mathrm{C}^{13}$ and $\delta \mathrm{C}^{14}$ values or the use of .95 oxalic acid as a base for age calculations. One point concerning the use of the oxalic-acid standard warrants discussion, however. As shown by Craig (1961), considerable differences in $\delta \mathrm{C}^{13}$ value exist between gases prepared by different laboratories. These differences presumably result from isotope fractionation during chemical conversion of the oxalic acid to the compound used for the radioactivity assay. If corrections are not made, the activity of standards used by various laboratories (and even by the same laboratory at different times) will not be identical. To avoid this, it is proposed that all laboratories correct the activity of counting gas (or liquid) prepared from oxalic acid to the activity it would have had if its $\delta \mathrm{C}^{13}$ value were -19.0. Thus,

$$
.95 \mathrm{~A}_{\mathrm{ox}}=.95 \mathrm{~A}^{\prime}{ }_{\mathrm{ox}}\left(1-\frac{2\left(19.0+\delta \mathrm{C}^{13{ }^{\prime}{ }_{\mathrm{ox}}}\right)}{1000}\right)
$$

where $\mathrm{A}^{\prime}{ }_{\text {ox }}$ and $\delta \mathrm{C}^{13}{ }^{\prime}{ }_{\text {ox }}$ are based on the actual counting and mass-spectrometer measurement made on a gas prepared from the oxalic-acid standard. If a further chemical conversion is required in order to convert the compound used for the radio assay into the compound (usually $\mathrm{CO}_{2}$ gas) used for mass analysis, this conversion, of course, must be carried out without any additional isotopic fractionation.

A recalibration of the mass-spectrometer used for $\mathrm{C}^{13}$ analyses at Lamont indicates that the previous calibration was in error over the entire range by 1.7 per mil. Thus a $\delta \mathrm{C}^{13}$ result given previously as -16.9 per mil would become -18.6. Although this change does not alter the relative values for any of the previously published Lamont results, it does introduce about a 3 per-mil error in comparing Lamont $\Delta \mathrm{C}^{14}$ results with those from other laboratories. Because of this, $\Delta$ values in this paper are expressed in terms of the new calibration and should supersede results published elsewhere. In order to correct the results in Lamont VI, 1.7 per mil should be subtracted from each $\delta \mathrm{C}^{13}$ value and 3.4 $\left(1+.001 \delta \mathrm{C}^{14}\right)$ should be added to each $\Delta \mathrm{C}^{14}$ value. Further conversion to the $\Delta$ scale requires the subtraction of $\frac{\delta \mathrm{C}^{14}}{20}$ from each result. Thus the results on the oxalic-acid standard (previously given as $\delta \mathrm{C}^{14}=53, \delta \mathrm{C}^{13}=$ -16.9 , and $\Delta \mathrm{C}^{14}=39 \pm 2$ ) become $\delta \mathrm{C}^{14}=53, \delta \mathrm{C}^{13}=-18.6, \Delta \mathrm{C}^{14}=42 \pm 2$ and $\Delta=39 \pm 2$. It should be noted that as the result of the change in calibration the $\delta \mathrm{C}^{13}$ for the Lamont oxalic-acid $\mathrm{CO}_{2}$ agrees within experimental error with that of -19.0 obtained by Craig (1961) for the same gas.

The $\mathrm{CO}_{2}$ method for measuring $\mathrm{C}^{14}$ activities was used in all cases. Details of the Lamont procedure have been published by Broecker, Tucek, and Olson (1959).

As the results on the samples in section IID have not been published elsewhere, a brief discussion of their significance is appropriate. Four types of information can be derived from these analyses; 1) the relationship between 
$\Delta$ for shells from open coastlines and $\Delta$ for surface water from the adjacent open ocean; 2) the magnitude of the Suess effect in the surface ocean; 3 ) the $\Delta$ value for areas of the surface ocean for which no direct measurements are available; 4) control values for age determinations on marine organisms.

The $\Delta$ values for coastal shells are compared with the average $\Delta$ values for the adjacent surface ocean in table 1 . As would be expected, the agreement is satisfactory, the mean deviation ( 11 per mil) being only slightly greater than that expected from the experimental errors.

\section{TABLE 1}

Comparison of $\Delta$ values for coastal shells with those for the adjacent surface ocean

\begin{tabular}{clccc}
\hline $\begin{array}{c}\text { Lamont } \\
\text { No. }\end{array}$ & \multicolumn{1}{c}{$\begin{array}{c}\text { Shell } \\
\text { Location }\end{array}$} & $\begin{array}{c}\text { Shell } \\
\text { Collection } \\
\text { Date }\end{array}$ & $\begin{array}{c}\Delta \\
\text { Shell }\end{array}$ & $\begin{array}{c}\text { Average } \\
\text { Adjacent } \\
\text { Open Ocean* }\end{array}$ \\
\hline L-576B & Bahamas & 1950 & $-51 \pm 5$ & -49 \\
L-593C & Bahamas & 1958 & $-25 \pm 7 \dagger$ & -49 \\
L-599A & Western France & 1952 & $-58 \pm 5$ & -49 \\
L-576A & Jamaica & 1930 & $-48 \pm 5$ & -51 \\
L-241A & Algeria & 1954 & $-43 \pm 10^{* *}$ & -49 \\
L-576E & Tahiti & 1957 & $-62 \pm 5$ & -49 \\
L-576C & Iceland & 1946 & $-64 \pm 6$ & $-54^{* * *}$ \\
\hline
\end{tabular}

* see Broecker and others, 1960

** see Broecker and Olson, 1959

*** based on the results of Fonselius and Östlund, 1959.

$\dagger$ the relatively high $\mathrm{C}^{14}$ concentration probably reflects the presence of bomb-produced C-14.

TABLE 2

Estimates of the Suess effect in the surface ocean

\begin{tabular}{lcccc}
\hline Location & $\begin{array}{c}\text { Lamont } \\
\text { No. }\end{array}$ & $\begin{array}{c}\text { Collection } \\
\text { Date }\end{array}$ & $\Delta$ & $\begin{array}{c}\text { Estimated } \\
\text { Suess Effect } \\
\text { in 1955 }\end{array}$ \\
\hline Jamaica & L-576F & 1884 & $-43 \pm 5$ & $13 \pm 17$ \\
Bahamas & L-576A & 1930 & $-48 \pm 5$ & \\
Iceland & L-576G & 1885 & $-55 \pm 7$ & $\leqslant 8$ \\
& L-576B & 1950 & $-51 \pm 5$ & \\
\multirow{3}{*}{ Tahiti } & L-576I & 1840 & $-72 \pm 6$ & \\
& L-576H & 1900 & $-69 \pm 6$ & $\leqslant 8$ \\
& L-576C & 1946 & $-64 \pm 6$ & \\
\hline
\end{tabular}

The magnitude of the Suess effect in the surface oceans has been estimated in four different localities by measuring pairs of gastropod shells collected at different times from each locality. The results as summarized in table 2 suggest that the decrease in $\Delta$ has been less than 10 per mil between 1880 
and 1955. Since the atmosphere showed a decrease in $\Delta$ of about 25 per mil over the same time interval, the rate of vertical mixing in the oceans must be quite rapid. This is in agreement with the vertical distribution of $\mathrm{Sr}^{90}$ in the oceans as observed by Bowen and Sugihara (1960). Rapid vertical mixing is also consistent with the fact that the observed atmospheric Suess effect is only a small fraction of what it would be if a major portion of combustion $\mathrm{CO}_{2}$ were not being taken into the ocean. A quantitative treatment of this problem will be published separately.

Very few surface-water samples were collected for radiocarbon analysis from the North Pacific prior to 1957. Because results on samples collected since that time are influenced by the presence of bomb-produced $\mathrm{C}^{14}$, measurements on shells grown before bomb-testing will perhaps be the only way to establish the steady-state $\Delta$ for these waters. Three of the samples reported here provide estimates of this value; Oahu, Hawaii (L-576J) $\Delta=-62 \pm 6$; Eniwetok Atoll (L-584A) $\Delta=-76 \pm 5$; and Vancouver Island (L-595) $\Delta=$ $-55 \pm 5$. Certainly many more such analyses are needed before any conclusions can be formulated.

The selection of a proper contemporary standard for radiocarbon dating of marine shells has always presented a serious problem. The results of this study suggest the following generalizations.

1) For age determinations on shells which formed on open-ocean coast lines in the latitude range from $40^{\circ} \mathrm{N}$ to $40^{\circ} \mathrm{S}$, a control value of $\Delta=-55 \pm$ 10 should be used. Since the $\mathrm{C}^{14}$ activity corresponding to this $\Delta$ value is almost the same as that of the usual standard for age determination of terrestrial organic materials (.95 oxalic acid), use of the latter standard seems justified for these shells. The additional error resulting from uncertainty in the initial $\mathrm{C}^{14} / \mathrm{C}^{12}$ ratio of mid-latitude shells will probably not increase the total error for such samples beyond that for terrestrial organic materials.

2) Because $\mathrm{C}^{14}$-deficient deep-water horizons outcrop at high latitude, age determination on samples formed there presents greater difficulty. This is particularly true in the Antarctic. As demonstrated by the measurement on the living seal from McMurdo Sound (L-570, this date list), marine organisms in this region form with an abnormally low $\mathrm{C}^{14}$ concentration, so that at death they appear to have ages as great as $1200 \mathrm{yr}$. Low values of $\mathrm{C}^{14}$ concentration are also found for surface water samples from the Antarctic. On the other hand, the effect in the northern North Atlantic should be much smaller, perhaps equivalent to an "initial age" below $200 \mathrm{yr}$. There are three reasons why this is so: a) the deep waters of the North Atlantic have relatively high $\Delta$ values (approximating-100 per mil), b) the Gulf Stream is a significant contributor to North Atlantic waters, and c) Atlantic circulation may be cyclic (Broecker and others, 1960).

3) The most difficult problem is the evaluation of results on samples from lagoons, estuaries and other restricted parts of the ocean. In these cases the contribution of limestone-derived carbon from terrestrial drainage may cause a significant depression in the initial $\Delta$. Whether this is the explanation for the low value of the Pearl Harbor sample (L-576D, this date list) is not certain; more probably, the result implies a finite age of the sample at the time of col- 
lection. More work is needed before the magnitude of these local effects can be evaluated.

\section{ACKNOWLEDGMENTS}

The authors were ably assisted in the laboratory by M. Zickl, J. Hubbard, F. Senn, N. Houston, and R. McPherson. Financial support was provided by the Division of Biology and Medicine of the Atomic Energy Commission (Contracts AT (30-1) 1808 and AT (30-1) 2493) and by the International Geophysical Year (grant Y/9.11/134).

\section{SAMPLE DESCRIPTIONS}

I. SAMPLES UTILIZING ATMOSPHERIC $\mathrm{CO}_{2}$

\section{A. Samples Defining the Atmospheric $C^{14} / C^{12}$ Ratio Before 1900}

\section{L-549A. Tokyo, Japan}

$$
\begin{array}{ccc}
\delta \mathrm{C}^{14} & \delta \mathrm{C}^{13} & \Delta \\
\mathbf{- 1} \pm \mathbf{5} & \mathbf{- 2 5 . 8} & \mathbf{1} \pm \mathbf{5}
\end{array}
$$

Wood from the 1849 to 1853 growth rings of a Quercus Glandulifera tree growing in a forest near Iwaizumi (Akita-Ken) Japan (39 $48^{\prime} \mathrm{N}$ Lat, $141^{\circ} 48^{\prime} \mathrm{E}$ Long). Coll. by K. Kigoshi, Gakushuin Univ., Tokyo.

\section{L-549B. Stockholm, Sweden}

Wood from the 1844, to 1856 growth rings of an oak tree from a resort area near Stockholm (59 $20^{\prime} \mathrm{N}$ Lat, $18^{\circ} 08^{\prime} \mathrm{E}$ Long). This wood has been used as a radiocarbon standard by the Stockholm Laboratory which obtained a $\Delta$ value of $6 \pm 5$ per mil. Subm. by G. Östlund for purposes of interlaboratory calibration.

B. Samples Defining the Atmospheric $C^{14} / C^{12}$ Ratio During the Time of the Suess Effect (1900-1952)

\section{L-458. Lower Hutt, New Zealand
Wood from pine lumber used by New Zea-} $-18 \pm 6-25.4-17 \pm 6$ land Laboratory as a standard (exact age unknown but cut prior to 1950). Subm. by T. Rafter and $\mathrm{G}$. Fergusson for purposes of an interlaboratory calibration. New Zealand Laboratory obtains a $\Delta$ value of $-18 \pm 4$ per mil.

\section{L-539B. Palisades, New York}

Wood from the 1952 growth ring of an oak tree cut April 19, 1959 on the grounds of Lamont Geol. Observatory $\left(41^{\circ} 00^{\prime} \mathrm{N}\right.$ Lat, $73^{\circ} 55^{\prime} \mathrm{W}$ Long). Sample was run to determine whether it contained carbon photosynthesized subsequent to formation of the ring. The $\Delta$ value is $25 \pm 10$ per

$2 \pm 6-23.7-1 \pm 6$ 
$\delta \mathrm{C}^{14} \quad \delta \mathrm{C}^{13} \quad \Delta$

mil greater than that for similar age rings from trees cut prior to 1954, suggesting that a small amount of bomb-produced $\mathrm{C}^{14}$ was incorporated into the ring subsequent to its formation. Also compare $\Delta$ for this sample with $-41 \pm 15$ for twigs coll. on Lamont grounds in 1952 (see L-184E, Broecker and Olson, 1959). The bulk sample was combusted without pretreatment. Coll. by the authors.

\section{Samples Defining the Atmospheric $C^{14} / C^{12}$ Ratio Since} Large-scale Bomb-testing Began (1952-1960)

\section{L-453. Stratosphere}

$225 \pm 6-7.2181 \pm 6$

Composite of several $\mathrm{CO}_{2}$ samples separated from stratospheric air, distributed by L. Machta, U. S. Weather Bureau, for purposes of intercalibration between the various laboratories interested in bomb-produced $\mathrm{C}^{14}$. Result obtained agrees well with those of the other participating laboratories.

L-493L. South Atlantic Ocean
Atmospheric $\mathrm{CO}_{2}$ coll. on Columbia Univ. Atmospheric $\mathrm{CO}_{2}$ coll. on Columbia Univ.
arch vessel $V$ ema by pulling air through $\mathrm{CO}_{2}$ free $\mathrm{KOH}\left(35^{\circ} \mathrm{S}\right.$ Lat, $24^{\circ} \mathrm{E}$ Long $)$. Coll. April 15 to 18,1958 by R. Gerard, Lamont Geol. Observatory (Broecker and Olson, 1960).

L493M. South Atlantic Ocean

$125 \pm 6 \quad-7.1 \quad 85 \pm 6$

Atmospheric $\mathrm{CO}_{2}$ coll. on Columbia Univ. research vessel Vema by pulling air through $\mathrm{CO}_{2}$ free $\mathrm{KOH}\left(27^{\circ} \mathrm{S}\right.$ Lat, $46^{\circ} \mathrm{E}$ Long). Coll. April 28 to May 2, 1958 by R. Gerard, Lamont Geol. Observatory (Broecker and Olson, 1960).

\section{L-493N. South Atlantic Ocean}

Atmospheric $\mathrm{CO}_{2}$ coll. on Columbia Univ. re$118 \pm 5 \quad-7.4 \quad 79 \pm 5$ earch vessel $V$ ema by pulling air through $\mathrm{CO}_{2}$ free $\mathrm{KOH}$ ( $13^{\circ} \mathrm{S}$ Lat, $65^{\circ} \mathrm{E}$ Long). Coll. May 13 to 17,1958 by R. Gerard, Lamont Geol. Observatory (Broecker and Olson, 1960).

\section{L-4930. Mediterranean Sea}

Atmospheric $\mathrm{CO}_{2}$ coll. on Columbia Univ. research vessel $V$ ema by pulling air through $\mathrm{CO}_{2}$ free $\mathrm{KOH}\left(36^{\circ} \mathrm{N}\right.$ Lat, $7^{\circ} \mathrm{E}$ Long $)$. Coll. August 8 to 10,1958 by R. Gerard, Lamont Geol. Observatory (Broecker and Olson, 1960). 


\section{L-493P. North Atlantic Ocean}

Atmospheric $\mathrm{CO}_{2}$ coll. on Columbia Univ. research vessel $V e m a$ by pulling air through $\mathrm{CO}_{2}$ free $\mathrm{KOH}\left(39^{\circ} \mathrm{N}\right.$ Lat, $30^{\circ} \mathrm{W}$ Long $)$. Coll. August 15 to 19,1958 by R. Gerard, Lamont Geol. Observatory (Broecker and Olson, 1960).

\section{L-487M. Santa Barbara, California}

Sycamore leaves from grounds of Santa Barbara Museum of Natural History $\left(34^{\circ} 31^{\prime} \mathrm{N}\right.$ Lat, $119^{\circ} 41^{\prime}$ W Long). Coll. September 9, 1958 by P. Orr.

\section{L529A. North Atlantic Ocean}

Atmospheric $\mathrm{CO}_{2}$ coll. on Columbia Univ. research vessel $V e m a$ by pulling air through $\mathrm{CO}_{2}$ free $\mathrm{KOH}\left(33^{\circ} \mathrm{N}\right.$ Lat, $76^{\circ} \mathrm{W}$ Long). Coll. October 22 to 24, 1958 by R. Gerard, Lamont Geol. Observatory (Broecker and Olson, 1960).

\section{L529M. North Atlantic Ocean}

Atmospheric $\mathrm{CO}_{2}$ coll. on Columbia Univ. research vessel $V$ ema by pulling air through $\mathrm{CO}_{2}$ free $\mathrm{KOH}\left(11^{\circ} \mathrm{N}\right.$ Lat, $76^{\circ} \mathrm{W}$ Long). Coll. November 8 to 10,1958 by R. Gerard, Lamont Geol. Observatory (Broecker and Olson, 1960).

\section{L537A. Equatorial Pacific Ocean}

Atmospheric $\mathrm{CO}_{2}$ coll. on Columbia Univ. research vessel $V e m a$ by pulling air through $\mathrm{CO}_{2}$ free $\mathrm{KOH}\left(1^{\circ} \mathrm{N}\right.$ Lat, $84^{\circ} \mathrm{W}$ Long $)$. Coll. December 1 to 4, 1958 by R. Gerard, Lamont Geol. Observatory (Broecker and Olson, 1960).

\section{L-528C. Palisades, New York}

Atmospheric $\mathrm{CO}_{2}$ coll. statically in $\mathrm{KOH}$ exposed $3 \mathrm{ft}$ above ground in dense forest on the grounds of the Lamont Geol. Observatory $\left(41^{\circ}\right.$ $00^{\prime} \mathrm{N}$ Lat, $73^{\circ} 55^{\prime} \mathrm{W}$ Long). Coll. by W. S. Broecker (Broecker and Olson, 1960).

\section{L-516E. Carson City, Nevada}

Willow leaves from tree growing $\mathrm{W}$ of town $\left(39^{\circ} 07^{\prime} \mathrm{N}\right.$ Lat, $119^{\circ} 53^{\prime} \mathrm{W}$ Long). Coll. June 5, 1959 by J. Calhoun, Nevada State Museum (Broecker and Olson, 1960).

$\begin{array}{ccc}\delta \mathrm{C}^{14} & \delta \mathrm{C}^{13} & \Delta \\ \mathbf{2 0 0} \pm \mathbf{8} & -7.5 & \mathbf{1 5 8} \pm \mathbf{8}\end{array}$

$109 \pm 6 \quad-25.6 \quad 110 \pm 6$

$197 \pm 5 \quad-6.8 \quad 153 \pm 5$

$193 \pm 8 \quad-8.3 \quad 153 \pm 8$

$176 \pm 9 \quad-7.8 \quad 136 \pm 9$

$264 \pm 7 \quad-18.6 \quad 248 \pm 7$

$249 \pm 8 \quad-25.5 \quad 250 \pm 8$ 
$\delta \mathrm{C}^{14} \quad \delta \mathrm{C}^{13} \quad \Delta$

L-528F. Rockleigh, New Jersey

$232 \pm 8 \quad-27.5 \quad 238 \pm 8$

Black-cherry leaves from tree growing on a golf course ( $41^{\circ} 00^{\prime} \mathrm{N}$ Lat, $73^{\circ} 58^{\prime} \mathrm{W}$ Long). Coll. June 9, 1959 by W. S. Broecker (Broecker and Olson, 1960).

\section{L-537L. South Pacific Ocean}

$144 \pm 7 \quad-8.9 \quad 107 \pm 7$

Atmospheric $\mathrm{CO}_{2}$ coll. on Columbia Univ. research vessel Vema by pulling air through $\mathrm{CO}_{2}$ free $\mathrm{KOH}\left(49^{\circ} \mathrm{S}\right.$ Lat, $75^{\circ} \mathrm{W}$ Long). Coll. January 28, 1959 by R. Gerard, Lamont Geol. Observatory (Broecker and Olson, 1960).

\section{L-519B. Lima, Peru}

$135 \pm 7 \quad-11.8 \quad 105 \pm 7$

Grass cut $\left(12^{\circ} \mathrm{S}\right.$ Lat, $77^{\circ} \mathrm{W}$ Long) March 6 , 1959 by O. C. Johnson, U. S. Atomic Energy Commission (Broecker and Olson, 1960. Note: the $\Delta \mathrm{C}^{14}$ given in this publication was incorrect).

L523. Tucson, Arizona

$193 \pm 9 \quad-22.4 \quad 187 \pm 8$

Leaves from tree growing in residential area on outskirts of Tucson $\left(32^{\circ} \mathrm{N}\right.$ Lat, $111^{\circ} \mathrm{W}$ Long). Coll. April 4, 1959 by W. S. Broecker (Broecker and Olson, 1960).

\section{L-528A. Palisades, New York}

Maple leaves from the grounds of Lamont Geol. Observatory $\left(41^{\circ} 00^{\prime} \mathrm{N}\right.$ Lat, $73^{\circ} 55^{\prime} \mathrm{W}$ Long). Coll. May 16, 1959 by W. S. Broecker (Broecker and Olson, 1960).

\section{L-528B. Alpine, New Jersey}

$251 \pm 6 \quad-20.6 \quad 240 \pm 6$

Atmospheric $\mathrm{CO}_{2}$ coll. statically in $\mathrm{KOH}$ on top of a $400-\mathrm{ft}$ radio tower $\left(40^{\circ} 57^{\prime} \mathrm{N}\right.$ Lat, $73^{\circ}$ $55^{\prime}$ W Long). Coll. May 16, to June 7, 1959 by the authors (Broecker and Olson, 1960).

\section{L-528E. Palisades, New York}

Maple leaves from the grounds of Lamont Geol. Observatory $\left(41^{\circ} 00^{\prime} \mathrm{N}\right.$ Lat, $73^{\circ} 55^{\prime} \mathrm{W}$ Long). Coll. June 7, 1959 by W. S. Broecker (Broecker and Olson, 1960).

L-547A. Alpine, New Jersey

$236 \pm 6 \quad-25.7 \quad 238 \pm 6$

Atmospheric $\mathrm{CO}_{2}$ coll. statically in $\mathrm{KOH}$ on top of a $400-\mathrm{ft}$ radio tower $\left(40^{\circ} 57^{\prime} \mathrm{N}\right.$ Lat, $73^{\circ}$ $55^{\prime}$ W Long). Coll. October 12 to 18,1959 by the authors.

$198 \pm 7 \quad-27.9205 \pm 7$ 


\section{L-547C. Alpine, New Jersey}

$\delta \mathrm{C}^{14} \quad \delta \mathrm{C}^{13} \quad \Delta$

Atmospheric $\mathrm{CO}_{2}$ coll. statically in $\mathrm{KOH}$ on top of a $400-\mathrm{ft}$ radio tower $\left(40^{\circ} 57^{\prime} \mathrm{N}\right.$ Lat, $73^{\circ}$ $55^{\prime} \mathrm{W}$ Long). Coll. October 25 to 28,1959 by the authors.

\section{L-547F. Alpine, New Jersey}

Atmospheric $\mathrm{CO}_{2}$ coll. statically in $\mathrm{KOH}$ on $220 \pm 6-26.0 \quad 222 \pm 6$ top of a $400-\mathrm{ft}$ radio tower $\left(40^{\circ} 57^{\prime} \mathrm{N}\right.$ Lat, $73^{\circ}$ $55^{\prime}$ W Long). Coll. November 25 to 28, 1959 by the authors.

D. Samples Defining the Amount of Bomb-produced $C^{14}$ in Humans

\section{L-371A. New York, New York $\quad 61 \pm 10 \quad-16.4 \quad 43 \pm 10$}

Human-lung tissue and associated blood from autopsy performed June 1958; subm. by A. Schulert.

\section{L-505A. Rockland County, New York}

Respiratory $\mathrm{CO}_{2}$ from a local resident, coll. January 1, 1959 by respiration into $\mathrm{CO}_{2}$-free $\mathrm{KOH}$ (Broecker, Olson and Schulert, 1959).

\section{L-505B. Rockland County, New York}

Blood from same person as L-505A; also coll. January 1, 1959.

\section{L-516A. Carson City, Nevada}

$118 \pm 7 \quad-18.1 \quad 103 \pm 7$

Blood from a local resident, coll. March 4, 1959; subm. by J. Calhoun, Nevada State Museum. (Broecker and Olson, 1960).

\section{L-519A. Lima, Peru}

Blood from a local resident, coll. March 6, 1959; subm. by O. C. Johnson (Broecker and Olson, 1960).

\section{L-516C. Ushuaia, Argentina}

Blood from a local resident, coll. March 15, $106 \pm 8 \quad-23.5 \quad 103 \pm 8$ 959 by M. Ewing, Lamont Geol. Observatory (Broecker and Olson, 1960).

\section{L-516D. Carson City, Nevada}

Blood from same person as L-516A, coll. $115 \pm 8 \quad-18.2 \quad 100 \pm 8$ $202 \pm 7 \quad-26.5206 \pm 7$ June 1, 1959; subm. by J. Calhoun (Broecker and Olson, 1960).

$$
33 \pm 8 \quad-20.9 \quad 25 \pm 8
$$

$135 \pm 6-19.7 \quad 123 \pm 6$ 


$$
\begin{array}{ccc}
\delta \mathrm{C}^{14} & \delta \mathrm{C}^{13} & \Delta \\
183 \pm 6 & -19.5 & 170 \pm 6
\end{array}
$$

L-516G. Carson City, Nevada

Blood from same person as L-516A, coll. December 17, 1959; subm. by J. Calhoun (Broecker and Olson, 1960).

L-569B. Rockland County, New York $213 \pm 6 \quad-19.9 \quad 201 \pm 6$

Respiratory $\mathrm{CO}_{2}$ from a local resident, coll. January 13, 1960 by breathing into large plastic bag (Broecker and Olson, 1960).

L-569A. Rockland County, New York $194 \pm 6 \quad-18.4 \quad 178 \pm 6$

Blood from same person as L-569B, coll. January 19, 1960 (Broecker and Olson, 1960).

L-583A. New York, New York $\quad 156 \pm 10 \quad-17.9 \quad 140 \pm 10$

Composite of blood-free muscle tissue coll. during an autopsy on February 8, 1960; subm. by P. Hudson, Columbia Univ. (Broecker and Olson, 1960).

E. Samples Defining the $C^{14}$ Concentration in Soil-Organic Materials

L-528D. Palisades, New York
$\mathrm{CO}_{2}$ given off by soil in dense forest on the

$\mathrm{CO}_{2}$ given off by soil in dense forest on the
ands of Lamont Geol. Observatory $\left(41^{\circ} 00^{\prime} \mathrm{N}\right.$ grounds of Lamont Geol. Observatory $73^{\circ} 55^{\prime} \mathrm{W}$ Long). Coll. continuously from May 16 to June 7, 1959 by exposing a tray of $\mathrm{CO}_{2}$-free $\mathrm{KOH}$ within an inverted barrel driven several in. into the soil. Total $\mathrm{CO}_{2}$ obtained indicated that 1.7 moles of $\mathrm{CO}_{2}$ were being given off per week from each square $m$ of soil surface (Broecker and Olson, 1960).

\section{L-528K. Palisades, New York
Coarse organic material from composite sam-} ple of soil (0 to 21 in.) coll. June 8, 1960 from same locality as L-528D. Sample, consisting of leaves, twigs, rootlets, etc., was obtained by sieving the soil through a window screen. Amount of material obtained corresponds to $4 \pm 2$ gm of carbon per kg of soil (Broecker and Olson, 1960).

\section{L-528L. Palisades, New York
Fine organic material from same soil a}

Fine organic material from same soil as L-528K. A representative sample of that portion
of the soil passing through the 40 -mesh screen was combusted. Amount of $\mathrm{CO}_{2}$ obtained corresponds to $10 \pm 2 \mathrm{gm}$ of carbon per $\mathrm{kg}$ of soil (Broecker and Olson, 1960).
$106 \pm 7 \quad-24.9 \quad 106 \pm 7$

$$
-1 \pm 7 \quad-26.1 \quad 1 \pm 7
$$

$-123 \pm 9-24.7-124 \pm 9$ 


\section{L-528G. Palisades, New York}

Humic acid extracted from fine fraction of soil (L-528L) by boiling it in $2 \% \mathrm{NaOH}$. Ca. $35 \%$ of the organics was dissolved by this treatment (Broecker and Olson, 1960).

\section{L-528P. Palisades, New York $\quad-218 \pm 9(-24.7)(-218 \pm 9)$}

Residual organics after treatment with

$\mathrm{NaOH}$. Ca. $55 \%$ of the organic material appeared in this fraction (Broecker and Olson, 1960).

\section{L-528R. Palisades, New York $\quad-83 \pm 20(-24.7)(-82 \pm 20)$}

Acid-soluble humic material from sample L-528L. That portion of the $\mathrm{NaOH}$-soluble organics which was not precipitated upon acidification of the $\mathrm{NaOH}$ with $\mathrm{HCl}$. Ca. $10 \%$ of the carbon in the fine fraction appeared in this fraction (Broecker and Olson, 1960).

\section{SAMPLES UTILIZING $\mathrm{CO}_{2}$ DisSOLVED IN SEA WATER}

A. $\mathrm{CO}_{2}$ obtained from acidified samples of sea water from Atlantic Ocean and adjacent seas. All samples in this group were coll. by the scientific staff of Columbia Univ. research vessel Vema, under direction of M. Ewing, R. Gerard, and B. Heezen. The techniques used in collecting and measuring samples have been published by Broecker, Tucek, and Olson (1959). The implications of the results have been discussed by Broecker, Gerard, Ewing, and Heezen (1960). The rather large variations in the $\delta \mathrm{C}^{13}$ ratios are the result of isotope fractionation during the processing of the sample and do not represent real variations within the ocean. The numbers in parentheses beside each laboratory number are the index numbers used by Broecker and others (1960). $\delta \mathrm{C}^{13}$ values in parenthises are estimated rather than measured. The corresponding $\Delta$ values are based on these estimates.

\section{1) Surface water 0 to $50 \mathrm{~m}$}

\section{L-326A. (1) Gulf Stream}

$-9 \pm 7 \quad 0.1 \quad-59 \pm 7$

Coll. October 30,$1955 ; 38^{\circ} 22^{\prime} \mathrm{N}$ Lat, $71^{\circ}$ $32^{\prime}$ W Long; $1 \mathrm{~m}$ depth.

L-287E. (2) North Atlantic Current $12 \pm 8$ (-3.7) (-31 \pm 10$)$

Coll. July 19,$1955 ; 37^{\circ} 58^{\prime} \mathrm{N}$ Lat, $50^{\circ} 53^{\prime}$

W Long; 1 m depth.

\section{L-326B. (3) Sargasso Sea}

Coll. November 1,$1955 ; 36^{\circ} 06^{\prime} \mathrm{N}$ Lat, $66^{\circ}$ 06 ' W Long; 1 m depth.

\section{L-538P. Gulf Stream}

Coll. July 10,$1959 ; 35^{\circ} 20^{\prime} \mathrm{N}$ Lat, $74^{\circ} 40^{\prime}$

$$
-5 \pm 6 \quad-2.8-49 \pm 6
$$

W Long; $1 \mathrm{~m}$ depth. 
L_326C. (4) Sargasso Sea

Coll. November 3,$1955 ; 34^{\circ} 06^{\prime} \mathrm{N}$ Lat, $65^{\circ}$ 06' W Long; $1 \mathrm{~m}$ depth.

L-465Z. (5) Sargasso Sea

Coll. November 13, 1957; $34^{\circ} 05^{\prime} \mathrm{N}$ Lat, $65^{\circ} 00^{\prime} \mathrm{W}$ Long; $1 \mathrm{~m}$ depth.

\section{L-367I. (6) Sargasso Sea}

Coll. June 2, 1956; $33^{\circ} 50^{\prime} \mathrm{N}$ Lat, $66^{\circ} 18^{\prime}$ W Long; $1 \mathrm{~m}$ depth.

\section{L-367K. (7) Sargasso Sea}

Coll. June 7,$1956 ; 33^{\circ} 00^{\prime} \mathrm{N}$ Lat, $49^{\circ} 48^{\prime} \mathrm{W}$ Long; $1 \mathrm{~m}$ depth.

\section{L-367J. (8) Sargasso Sea}

Coll. June 5 , $1956 ; 32^{\circ} 38^{\prime} \mathrm{N}$ Lat, $57^{\circ} 55^{\prime} \mathrm{W}$ Long; $1 \mathrm{~m}$ depth.

L-367L. (9) Sargasso Sea

Coll. June 12, $1956 ; 31^{\circ} 45^{\prime} \mathrm{N}$ Lat, $34^{\circ} 38^{\prime}$

$$
\begin{array}{ccc}
\delta \mathrm{C}^{14} & \delta \mathrm{C}^{13} & \Delta \\
4 \pm 6 & (3.3) & (-53 \pm 8)
\end{array}
$$$$
-1 \pm 5 \quad-0.2-51 \pm 5
$$$$
-5 \pm 5 \quad 0.5 \quad-56 \pm 5
$$$$
-6 \pm 6 \quad-1.0 \quad-55 \pm 6
$$

$$
9 \pm 6-0.3-41 \pm 6
$$
$-13 \pm 7(-0.7)(-62 \pm 10)$ W Long; $1 \mathrm{~m}$ depth.

L-464B. (10) Sargasso Sea

Coll. November 16, 1957; $29^{\circ} 57^{\prime} \mathrm{N}$ Lat, $61^{\circ} 41^{\prime}$ W Long; $1 \mathrm{~m}$ depth.

L-464D. (11) Sargasso Sea

Coll. November 17, 1957; $29^{\circ} 13^{\prime} \mathrm{N}$ Lat, $60^{\circ} 30^{\prime} \mathrm{W}$ Long; $46 \mathrm{~m}$ depth.

\section{L-326D. (12) Antilles Current}

Coll. November 5,$1955 ; 27^{\circ} 05^{\prime} \mathrm{N}$ Lat, $73^{\circ}$ $32^{\prime} \mathrm{W}$ Long; $1 \mathrm{~m}$ depth.

\section{L-416A. (13) Florida Current}

Coll. June 24, 1957; $25^{\circ} 42^{\prime}$ N Lat, $79^{\circ} 23^{\prime}$

$$
26 \pm 5 \quad(3.3)(-32 \pm 8)
$$

W Long; $1 \mathrm{~m}$ depth.

\section{L-326E. (14) Antilles Current}

Coll. November 8, $1955 ; 25^{\circ} 25^{\prime} \mathrm{N}$ Lat, $75^{\circ}$ 13' W Long; $1 \mathrm{~m}$ depth.

\section{L-326G. (15) Antilles Current}

Coll. November 12, 1955; $20^{\circ} 32^{\prime} \mathrm{N}$ Lat, $68^{\circ} 30^{\prime} \mathrm{W}$ Long; $1 \mathrm{~m}$ depth.

\section{L-326F.(16) Antilles Current \\ Coll. November 10, 1955; $19^{\circ} 58^{\prime} \mathrm{N}$ Lat, $70^{\circ} 53^{\prime} \mathrm{W}$ Long; $1 \mathrm{~m}$ depth.} $13 \pm 6 \quad-3.5 \quad 31 \pm 6$$$
5 \pm 6 \quad 3.2 \quad-51 \pm 6
$$$$
\begin{array}{llll}
-2 \pm 7 & 0.6 & -49 \pm 7
\end{array}
$$$$
6 \pm 5 \quad-0.9 \quad-42 \pm 5
$$ 


\section{L-326H. (17) Antilles Current}

Coll. November 14,$1955 ; 19^{\circ} 07^{\prime} \mathrm{N}$ Lat,

$$
\begin{array}{ccc}
\delta \mathrm{C}^{14} & \delta \mathrm{C}^{13} & \Delta \\
\mathbf{8} \pm \mathbf{5} & \mathbf{0 . 4} & \mathbf{- 4 3} \pm \mathbf{5}
\end{array}
$$

$67^{\circ} 07^{\prime} \mathrm{W}$ Long; $1 \mathrm{~m}$ depth.

\section{L-529I. (165) West Caribbean}

Coll. November 6,$1958 ; 15^{\circ} 51^{\prime} \mathrm{N}$ Lat, $75^{\circ}$

$20 \pm 7 \quad 2.1 \quad-35 \pm 7$

11' W Long; $1 \mathrm{~m}$ depth.

\section{L-4640. (18) North Equatorial Current}

Coll. November 26, 1957; $15^{\circ} 03^{\prime} \mathrm{N}$ Lat, $39^{\circ} 48^{\prime}$ W Long; 1 m depth.

\section{L-538I. (163) North Equatorial Current}

Coll. May 14, $1959 ; 10^{\circ} 31^{\prime} \mathrm{N}$ Lat, $45^{\circ} 02^{\prime}$ W Long; 1 m depth.

\section{L-326R. (19) West Caribbean}

Coll. December 20,$1955 ; 19^{\circ} 04^{\prime} \mathrm{N}$ Lat, $80^{\circ}$ 48' W Long; $1 \mathrm{~m}$ depth.

\section{L-326P. (20) West Caribbean}

Coll. December 10, 1955; $16^{\circ} 17^{\prime} \mathrm{N}$ Lat, $79^{\circ}$ 14' W Long; $1 \mathrm{~m}$ depth.

\section{L-326Q.(21) West Caribbean}

Coll. December 16, 1955; $17^{\circ} 39^{\prime} \mathrm{N}$ Lat, $79^{\circ}$ 04' W Long; $1 \mathrm{~m}$ depth.

\section{L-3260.(22) East Caribbean}

Coll. December 8, $1955 ; 12^{\circ} 27^{\prime} \mathrm{N}$ Lat, $77^{\circ}$ $25^{\prime}$ W Long; 1 m depth.

\section{L-326L. (23) East Caribbean}

Coll. November 25,$1955 ; 17^{\circ} 05^{\prime} \mathrm{N}$ Lat, $71^{\circ}$ $36^{\prime}$ W Long; 1 m depth.

\section{L-326J. (24) East Caribbean}

Coll. November 19, $1955 ; 16^{\circ} 43^{\prime} \mathrm{N}$ Lat, $70^{\circ}$ $38^{\prime}$ W Long; 1 m depth.

\section{L-326I. (25) East Caribbean}

Coll. November 17,$1955 ; 17^{\circ} 11^{\prime} \mathrm{N}$ Lat, $68^{\circ}$

$$
1 \pm 8 \quad-2.7 \quad-44 \pm 8
$$

$19 \pm 6 \quad 2.4 \quad-37 \pm 6$

$$
-1 \pm 7(1.3)(-52 \pm 9)
$$

$$
-11 \pm 10 \quad 5.5 \quad-71 \pm 10
$$

$$
-5 \pm 7 \quad-2.8-49 \pm 7
$$

$$
9 \pm 6 \quad 0.6 \quad-43 \pm 6
$$

$$
9 \pm 7 \quad 3.8 \quad-49 \pm 7
$$

$$
\begin{array}{lll}
4 \pm 7 & 2.9 & -52 \pm 7
\end{array}
$$

$$
-1 \pm 6 \quad 3.4 \quad-58 \pm 6
$$
55' W Long; 1 m depth.

\section{L-326K.(26) East Caribbean}

Coll. November 22,$1955 ; 17^{\circ} 47^{\prime} \mathrm{N}$ Lat, $68^{\circ}$

$$
11 \pm 7 \quad 4.2 \quad-48 \pm 7
$$
$22^{\prime} \mathrm{W}$ Long; $1 \mathrm{~m}$ depth.

\section{L-367Y.(27) East Mediterranean \\ Coll. August 16, 1956;34 $04^{\circ} \mathrm{N}$ Lat, $26^{\circ}$}

$$
\begin{array}{rrr}
-4 \pm 7 & 2.5 & -59 \pm 7
\end{array}
$$




\section{L-367R. (28) Gulf of Corinth \\ $\delta \mathrm{C}^{14} \quad \delta \mathrm{C}^{13} \quad \Delta$ \\ Coll. July 24, $1956 ; 38^{\circ} 06^{\prime} \mathrm{N}$ Lat, $22^{\circ} 54^{\prime}$ \\ E Long; $1 \mathrm{~m}$ depth.}

L-464R. (29) South Equatorial Current $\quad-28 \pm 6 \quad-2.8 \quad-71 \pm 6$ Coll. December 5, 1957; $00^{\circ} 51^{\prime} \mathrm{N}$ Lat, $32^{\circ}$

$52^{\prime} \mathrm{W}$ Long; $49 \mathrm{~m}$ depth. L-334A. (30) South Equatorial Current
Coll. February 15,$1956 ; 03^{\circ} 06^{\prime}$ S Lat, $32^{\circ}$

$26^{\prime} \mathrm{W}$ Long; $1 \mathrm{~m}$ depth.

L-334E. (31) South Equatorial Current $-13 \pm 7(-0.7)(-61 \pm 10)$

Coll. February 17, $1956 ; 03^{\circ} 34^{\prime} \mathrm{S}$ Lat, $31^{\circ}$

$22^{\prime}$ W Long; 1 m depth.

L-410D. (32) South Equatorial Current $\quad-24 \pm 5 \quad-2.1 \quad-69 \pm 5$

Coll. January 9,$1957 ; 04^{\circ} 29^{\prime} \mathrm{S}$ Lat, $34^{\circ} 54^{\prime}$

W Long; 1 m depth.

L-419B. (33) North Benguela Current $\quad-24 \pm 7 \quad-5.0 \quad-63 \pm 7$

Coll. May 20, $1957 ; 05^{\circ} 41^{\prime} \mathrm{S}$ Lat, $10^{\circ} 39^{\prime}$ E Long; $1 \mathrm{~m}$ depth.

\section{L-464T. (34) Brazil Current \\ Coll. December 16, 1957; $09^{\circ} 38^{\prime} \mathrm{S}$ Lat, $34^{\circ}$}

$$
-8 \pm 7(-2.7)(-52 \pm 9)
$$

05' W Long; $1 \mathrm{~m}$ depth.

L-410E. (35) Brazil Current
Coll. January 19, 1957; $10^{\circ} 59^{\prime}$ S Lat, $32^{\circ}$

28' W Long; $1 \mathrm{~m}$ depth.

$$
\begin{array}{llll}
\text { L-465D. (36) Brazil Current } & \mathbf{7} \pm \mathbf{7} & \mathbf{- 3 . 8} & \mathbf{3 6} \pm \mathbf{7}
\end{array}
$$

$38^{\prime} \mathrm{W}$ Long; $1 \mathrm{~m}$ depth.

L-410JJ. (37) Central South Atlantic $\quad-10 \pm 15(-2.7)(-54 \pm 15)$

Coll. May 12,$1957 ; 14^{\circ} 30^{\prime} \mathrm{S}$ Lat, $07^{\circ} 34^{\prime}$

E Long; $1 \mathrm{~m}$ depth.

L-410DD. (39) Central South Atlantic $-13 \pm 5 \quad-3.0 \quad-56 \pm 5$

Coll. May 3,$1957 ; 25^{\circ} 31^{\prime} \mathrm{S}$ Lat, $12^{\circ} 26^{\prime} \mathrm{E}$

Long; $1 \mathrm{~m}$ depth.

L-410AA. (40) Central South Atlantic
Coll. April 29,$1957 ; 32^{\circ} 13^{\prime}$ S Lat, $16^{\circ} 20^{\prime}$

E Long; $1 \mathrm{~m}$ depth.

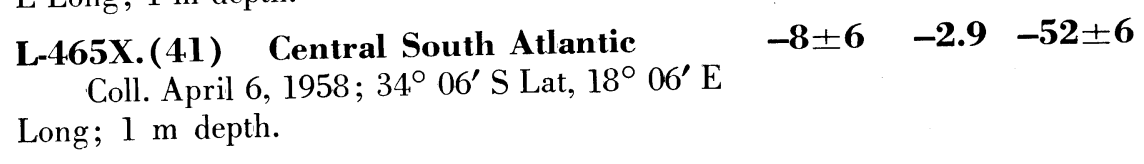




\section{L-410Y. (42) West Benguela \\ Coll. April 19, 1957; $34^{\circ} 46^{\prime} \mathrm{S}$ Lat, $06^{\circ} 29^{\prime}$

$$
\begin{array}{ccc}
\delta \mathrm{C}^{14} & \delta \mathrm{C}^{13} & \Delta \\
\mathbf{- 2} \pm \mathbf{7} & \mathbf{- 6 . 8} & \mathbf{- 3 8} \pm \mathbf{7}
\end{array}
$$

E Long; 1 m depth.

\section{L-410S. (43) West Wind Drift}

Coll. April 6, 1957; $39^{\circ} 03^{\prime} \mathrm{S}$ Lat, $41^{\circ} 48^{\prime}$

$$
-2 \pm 5 \quad-0.7 \quad-51 \pm 5
$$

W Long; $1 \mathrm{~m}$ depth.

\section{L-410W. (44) West Wind Drift}

Coll. April 12, 1957; 40 $54^{\prime}$ S Lat, $20^{\circ} 29^{\prime}$

$$
-21 \pm 7 \quad-8.3 \quad-54 \pm 7
$$

W Long; 1 m depth.

\section{L-410X. (45) West Wind Drift}

Coll. April 15, 1957; $41^{\circ} 15^{\prime} \mathrm{S}$ Lat, $06^{\circ} 10^{\prime}$

$$
-14 \pm 7 \quad-3.9 \quad-56 \pm 7
$$

W Long; $1 \mathrm{~m}$ depth.

\section{L-410Q. (46) Falkland Current}

Coll. April 2, 1957; $40^{\circ} 43^{\prime} \mathrm{S}$ Lat, $56^{\circ} 32^{\prime}$

$$
-27 \pm 5 \quad-1.1 \quad-74 \pm 5
$$

W Long; 1 m depth.

\section{L-410R. (47) Falkland Current}

Coll. April 3, 1957; $41^{\circ} 05^{\prime} \mathrm{S}$ Lat, $51^{\circ} 09^{\prime}$

$$
-27 \pm 7 \quad-3.3 \quad-69 \pm 7
$$

W Long; $1 \mathrm{~m}$ depth.

\section{Lr410P.(48) Falkland Current}

Coll. March 20, 1957; 45 $24^{\prime}$ S Lat, $59^{\circ} 13^{\prime}$

$$
-27 \pm 6 \quad-1.4 \quad-73 \pm 6
$$

W Long; 1 m depth.

\section{L-465S. (49) Antaretic Convergence}

Coll. March 24, 1958; $51^{\circ} 27^{\prime} \mathrm{S}$ Lat, $02^{\circ} 38^{\prime}$

$$
-28 \pm 6 \quad 3.9-84 \pm 6
$$

E Long, $1 \mathrm{~m}$ depth.

\section{L-465M. (50) Drake Passage}

Coll. February 24,$1958 ; 55^{\circ} 27^{\prime} \mathrm{S}$ Lat, $57^{\circ}$

$$
-69 \pm 7 \quad-5.9-105 \pm 7
$$

$10^{\prime} \mathrm{W}$ Long; $1 \mathrm{~m}$ depth.

\section{L-465R.(51) Antaretic Surface Water $\quad-79 \pm 5 \quad-6.6-113 \pm 5$}

Coll. March 19, 1958; $57^{\circ} 07^{\prime} \mathrm{S}$ Lat, $07^{\circ}$

15' W Long; $1 \mathrm{~m}$ depth.

$$
\text { 2) Subsurface water } 200 \text { to } 400 \mathrm{~m}
$$

\section{L-334U. (52) North Atlantic Central Water $-11 \pm 7 \quad 1.2 \quad-63 \pm 7$}

Coll. April 16, $1956 ; 25^{\circ} 01^{\prime} \mathrm{N}$ Lat, $59^{\circ} 12^{\prime}$

W Long; $256 \mathrm{~m}$ depth.

\section{L-367EE. (53) North Atlantic Central Water}

Coll. October 12,$1956 ; 23^{\circ} 22^{\prime} \mathrm{N}$ Lat, $27^{\circ} \mathbf{- 1 5}^{\mathbf{- 1 5}} \quad \mathbf{2 . 5}-\mathbf{6 9} \pm \mathbf{5}$ 14' W Long; $329 \mathrm{~m}$ depth. 
L-334M. (54) North Atlantic Central Water

$\delta \mathrm{C}^{14} \quad \delta \mathrm{C}^{13} \quad \Delta$

Coll. April 11, 1956; $15^{\circ} 49^{\prime} \mathrm{N}$ Lat, $47^{\circ} 12^{\prime}$

$-29 \pm 6 \quad-4.3-69 \pm 6$

W Long; $229 \mathrm{~m}$ depth.

L-334K. (55) Transition NACW-SACW $\quad-49 \pm 9 \quad-4.6 \quad-88 \pm 9$

Coll. April 7, 1956; $08^{\circ} 15^{\prime} \mathrm{N}$ Lat, $37^{\circ} 54^{\prime}$

W Long; 274 m depth.

L-464P.(56) Transition NACW-SACW $-41 \pm 6 \quad-2.6 \quad-84 \pm 6$

Coll. December 3, 1957; $07^{\circ} 22^{\prime} \mathrm{N}$ Lat, $29^{\circ}$

$59^{\prime} \mathrm{W}$ Long; $240 \mathrm{~m}$ depth.

L-419G. (57) South Atlantic Central Water

Coll. June 9, 1957;00 $10^{\prime} \mathrm{S}$ Lat, $24^{\circ} 29^{\prime}$

$-18 \pm 7 \quad-4.1 \quad-59 \pm 7$

W Long; $201 \mathrm{~m}$ depth.

L-334B. (58) South Atlantic Central Water

Coll. February 16,$1956 ; 03^{\circ} 17^{\prime} \mathrm{S}$ Lat, $32^{\circ}$

$$
-35 \pm 7 \quad-2.1 \quad-79 \pm 7
$$

14' W Long; 274 m depth.

L-419A. (59) Lower SACW $\quad \mathbf{- 3 8} \pm \mathbf{7} \quad \mathbf{- 4 . 2} \quad \mathbf{- 7 8} \pm \mathbf{7}$

Coll. May 19, $1957 ; 06^{\circ} 25^{\prime} \mathrm{S}$ Lat, $11^{\circ} 26^{\prime}$

W Long; $366 \mathrm{~m}$ depth.

L-464Y.(60) South Atlantic Central Water $-8 \pm 5 \quad-3.6 \quad-50 \pm 5$

Coll. December 21, 1957; $14^{\circ} 02^{\prime} \mathrm{S}$ Lat, $37^{\circ}$

32 ' W Long; $295 \mathrm{~m}$ depth.

L-464Z.(61) South Atlantic Central Water $-4 \pm 6 \quad-0.1 \quad-54 \pm 6$

Coll. December 21, 1957; $14^{\circ} 10^{\prime} \mathrm{S}$ Lat, $37^{\circ}$

$33^{\prime}$ W Long; $300 \mathrm{~m}$ depth.

\section{L-410HH.(62) South Atlantic Central Water}

Coll. May 9,$1957 ; 21^{\circ} 14^{\prime} \mathrm{S}$ Lat, $03^{\circ} 22^{\prime} \mathrm{E}$

$$
-13 \pm 7 \quad-3.2-56 \pm 7
$$

Long. 0-274 m depth.

L-410BB.(63) South Atlantic Central Water

Coll. May 2, $1957 ; 28^{\circ} 26^{\prime} \mathrm{S}$ Lat, $08^{\circ} 39^{\prime} \mathrm{E}$

$$
-4 \pm 7 \quad-4.2-45 \pm 7
$$

Long; $201 \mathrm{~m}$ depth.

3) Subsurface water 500 to $1200 \mathrm{~m}$

\section{L-287G. (64) Lower North Atlantic Central Water}

Coll. July 19,$1955 ; 38^{\circ} 00^{\prime} \mathrm{N}$ Lat, $51^{\circ} 37^{\prime}$

$7 \pm 13(-3.7)(-35 \pm 13)$

W Long; $732 \mathrm{~m}$ depth. 


\section{L-367CC. (65) North Atlantic Undefined}

$\delta \mathrm{C}^{14} \quad \delta \mathrm{C}^{13} \quad \Delta$

Coll. October 11, 1956; $24^{\circ} 23^{\prime} \mathrm{N}$ Lat, $24^{\circ}$

$$
-41 \pm 10 \quad 6.8-102 \pm 10
$$

03' W Long; $1097 \mathrm{~m}$ depth.

\section{L-282M.(66) North Atlantic Undefined}

Coll. June 17, $1955 ; 23^{\circ} 28^{\prime} \mathrm{N}$ Lat $65^{\circ} \mathbf{5 6}^{\prime} \pm \mathbf{1 3}(\mathbf{- 3 . 7})(\mathbf{- 9 0} \pm \mathbf{1 3})$

W Long; 896 m depth.

L-3340. (67) North Atlantic Undefined
Coll. April 14, $1956 ; 22^{\circ} 22^{\prime}$ N Lat, $54^{\circ} 19^{\prime}$ W Long; $823 \mathrm{~m}$ depth.

L-464H.(68) North Atlantic Undefined $\quad-57 \pm 6 \quad-16.5 \quad-73 \pm 6$ Coll. November 22,$1957 ; 20^{\circ} 44^{\prime} \mathrm{N}$ Lat, $49^{\circ}$ 24' W Long; $950 \mathrm{~m}$ depth.

L-326U.(69) North Atlantic Undefined $\quad-43 \pm 7 \quad-3.0 \quad-85 \pm 7$ Coll. December 25, 1955; $19^{\circ} 09^{\prime} \mathrm{N}$ Lat, $76^{\circ}$ 59' W Long; 1097 m depth.

L-334L. (70) North Atlantic Undefined $-50 \pm 6 \quad-1.7 \quad-94 \pm 6$ Coll. April 11, 1956; $15^{\circ} 49^{\prime} \mathrm{N}$ Lat, $47^{\circ} 12^{\prime}$

W Long; $823 \mathrm{~m}$ depth.

L-464M. (71) North Atlantic Undefined $-73 \pm 6 \quad-8.8-103 \pm 6$ Coll. November 26, $1957 ; 15^{\circ} 29^{\prime} \mathrm{N}$ Lat, $40^{\circ}$ $30^{\prime}$ W Long; 823 m depth.

L-334I. (72) North Atlantic Undefined $-60 \pm 5-1.8-104 \pm 5$ Coll. April 7, 1956; $08^{\circ} 14^{\prime} \mathrm{N}$ Lat, $37^{\circ} 48^{\prime}$ W Long; $823 \mathrm{~m}$ depth.

L-464S. (73) Antarctic Intermediate Water

Coll. December 5, 1957; $00^{\circ} 51^{\prime} \mathrm{N}$ Lat, $32^{\circ}$ $-63 \pm 6 \quad-3.0-104 \pm 6$ 52' W Long; $799 \mathrm{~m}$ depth.

L-334C. (74) Antarctic Intermediate Water

Coll. February 16,$1956 ; 03^{\circ} 22^{\prime} \mathrm{S}$ Lat, $31^{\circ}$ $-76 \pm 6 \quad-1.9-119 \pm 6$ $50^{\prime} \mathrm{W}$ Long; $732 \mathrm{~m}$ depth.

\section{L-419C.(75) Antarctic Intermediate Water}

Coll. May 26, $1957 ; 04^{\circ} 47^{\prime} \mathrm{S}$ Lat, $02^{\circ} 40^{\prime} \mathrm{E}$ $-64 \pm 7 \quad-14.4-84 \pm 7$ Long; $732 \mathrm{~m}$ depth. 
$\begin{array}{llll}\delta \mathrm{C}^{14} & \delta \mathrm{C}^{13} & \Delta\end{array}$

L-464U.(76) Antarctic Intermediate Water

Coll. December 16, $1957 ; 10^{\circ} 04^{\prime} \mathrm{S}$ Lat, $33^{\circ}$

$$
-86 \pm 6 \quad-2.4-127 \pm 6
$$

52 ' W Long; 914 m depth.

L-410II. (77) Upper Antarctic Intermediate Water

Coll. May 9, $1957 ; 21^{\circ} 09^{\prime} \mathrm{S}$ Lat, $03^{\circ} 20^{\prime} \mathrm{E}$

$$
-67 \pm 7 \quad-3.8-107 \pm 9
$$

Long; $550 \mathrm{~m}$ depth.

L-410CC. (78) Lower Antarctic Intermediate Water

Coll. May 2, $1957 ; 28^{\circ} 25^{\prime} \mathrm{S}$ Lat, $08^{\circ} 36^{\prime} \mathrm{E}$

$$
-92 \pm 7 \quad-4.1-130 \pm 7
$$

Long; $1189 \mathrm{~m}$ depth.

4) Subsurface samples 1200 to $2500 \mathrm{~m}$

L-450E.(80) Upper North Atlantic Deep Water

Coll. October 31, 1957; $39^{\circ} 16^{\prime} \mathrm{N}$ Lat, $70^{\circ}$

$$
-22 \pm 5 \quad-3.9 \quad-63 \pm 5
$$

46' W Long; $2323 \mathrm{~m}$ depth.

\section{L-287F. (81) Upper North Atlantic Deep Water}

Coll. July 19,$1955 ; 37^{\circ} 58^{\prime} \mathrm{N}$ Lat, $50^{\circ} 53^{\prime}$

$$
-19 \pm 7(-3.7)(-62 \pm 13)
$$

W Long; 1944 m depth.

L-464E. (82) Upper North Atlantic Deep Water

Coll. November 17, 1957; $29^{\circ} 13^{\prime} \mathrm{N}$ Lat,

$60^{\circ} 31^{\prime}$ W Long; $1423 \mathrm{~m}$ depth.

L-282K. (83) Upper North Atlantic Deep Water

Coll. June 15,$1955 ; 25^{\circ} 07^{\prime} \mathrm{N}$ Lat, $69^{\circ} 57^{\prime}$

$$
-32 \pm 10(-4.7)(-74 \pm 10)
$$

Long; $1829 \mathrm{~m}$ depth.

\section{L-538M. (158) Upper North Atlantic Deep Water}

Coll. May 30, 1959; $21^{\circ} 10^{\prime} \mathrm{N}$ Lat, $66^{\circ} 37^{\prime}$

$$
-59 \pm 7(-0.7)(-105 \pm 7)
$$

W Long; $2520 \mathrm{~m}$ depth.

L-529C. (84) Upper North Atlantic Deep Water

Coll. November 4, 1957; $20^{\circ} 31^{\prime} \mathrm{N}$ Lat, $73^{\circ}$

$$
-5 \pm 6 \quad-4.4 \quad-46 \pm 6
$$

$22^{\prime}$ W Long; $1230 \mathrm{~m}$ depth.

L-529E. (152) Upper North Atlantic Deep Water

Coll. November 4,$1958 ; 20^{\circ} 31^{\prime} \mathrm{N}$ Lat, $73^{\circ}$

$$
\begin{array}{lll}
-9 \pm 7 & 0.3 & -59 \pm 7
\end{array}
$$

22' W Long; 1783 m depth. 


\section{L-529D. (154) Upper North Atlantic Deep Water}

$$
\delta \mathrm{C}^{14} \quad \delta \mathrm{C}^{13} \quad \Delta
$$

Coll. November 4,$1958 ; 20^{\circ} 31^{\prime} \mathrm{N}$ Lat, $73^{\circ}$

$$
-34 \pm 6 \quad 0.7 \quad-84 \pm 6
$$

$22^{\prime}$ W Long; $1863 \mathrm{~m}$ depth.

\section{L-529F. (160) Upper North Atlantic Deep Water}

Coll. November 5,$1958 ; 19^{\circ} 31^{\prime} \mathrm{N}$ Lat, $74^{\circ}$

$$
-32 \pm 6 \quad-2.8 \quad-75 \pm 6
$$

$59^{\prime}$ W Long; $1290 \mathrm{~m}$ depth.

\section{L-538L. (155) Upper North Atlantic Deep Water}

Coll. May 25, 1959; $18^{\circ} 58^{\prime} \mathrm{N}$ Lat, $65^{\circ} 38^{\prime}$

$$
-30 \pm 6 \quad 0.2-79 \pm 6
$$

W Long; $1260 \mathrm{~m}$ depth.

\section{L-419J. (87) Upper North Atlantic Deep Water}

Coll. July 4,$1957 ; 11^{\circ} 12^{\prime} \mathrm{N}$ Lat, $59^{\circ} 19^{\prime} \mathrm{W}$

$$
-61 \pm 7 \quad-14.7-80 \pm 7
$$

Long; $2305 \mathrm{~m}$ depth.

\section{L-419I. (88) Upper North Atlantic Deep Water}

Coll. July 4,$1957 ; 11^{\circ} 12^{\prime} \mathrm{N}$ Lat, $57^{\circ} 16^{\prime} \mathrm{W}$

$$
-43 \pm 6 \quad-7.3 \quad-77 \pm 6
$$

Long; $1765 \mathrm{~m}$ depth.

\section{L-419H. (89) Upper North Atlantic Deep Water}

Coll. July 3,$1957 ; 10^{\circ} 49^{\prime} \mathrm{N}$ Lat, $55^{\circ} 4 \mathrm{l}^{\prime} \mathrm{W}$

$$
-62 \pm 6-17.6-76 \pm 6
$$

Long; $1262 \mathrm{~m}$ depth.

\section{L-367BB. (90) Upper North Atlantic Deep Water}

Coll. October 10, 1956; $25^{\circ} 13^{\prime} \mathrm{N}$ Lat, $21^{\circ}$

$$
\begin{array}{lll}
-56 \pm 5 & -2.4 & -99 \pm 5
\end{array}
$$

$23^{\prime}$ W Long; 1463 m depth.

\section{L-367AA. (91) Upper North Atlantic Deep Water}

Coll. September 10, 1956; $25^{\circ} 13^{\prime} \mathrm{N}$ Lat,

$$
-60 \pm 6 \quad-1.4-104 \pm 6
$$
$21^{\circ} 23^{\prime} \mathrm{W}$ Long; $1829 \mathrm{~m}$ depth.

\section{L-334J.(92) Upper North Atlantic Deep Water

$$
-53 \pm 7 \quad 0.8-102 \pm 7
$$

Coll. April 7, 1956; $08^{\circ} 15^{\prime} \mathrm{N}$ Lat, $37^{\circ} 53^{\prime}$

W Long; 1463 m depth.

\section{L-419D.(93) Upper North Atlantic Deep Water}

Coll. May 27, 1957; $04^{\circ} 23^{\prime} \mathrm{S}$ Lat, $00^{\circ} 05^{\prime}$

$$
-47 \pm 6 \quad-2.5 \quad-90 \pm 6
$$

W Long; $1829 \mathrm{~m}$ depth. 


\section{L-464W (94) Upper North Atlantic Deep Water

$$
\delta \mathrm{C}^{14} \quad \delta \mathrm{C}^{13} \quad \Delta
$$

$$
-54 \pm 8 \quad-2.6 \quad-96 \pm 8
$$

Coll. December 16, 1957; $10^{\circ} 06^{\prime} \mathrm{S}$ Lat, $34^{\circ}$

54' W Long; $1829 \mathrm{~m}$ depth.

\section{L-464X (95) Upper North Atlantic Deep Water}

Coll. December 17, $1957 ; 12^{\circ} 37^{\prime} \mathrm{S}$ Lat, $35^{\circ}$

$$
-55 \pm 9-8.3-87 \pm 9
$$
$00^{\prime} \mathrm{W}$ Long; $1829 \mathrm{~m}$ depth.

\section{L-465B (96) Upper North Atlantic Deep Water}

Coll. December 22,$1957 ; 15^{\circ} 17^{\prime} \mathrm{S}$ Lat, $36^{\circ}$

$$
-61 \pm 7-1.3-106 \pm 7
$$
$00^{\prime} \mathrm{W}$ Long; $4369 \mathrm{~m}$ depth.

L-410GG. (97) Antarctic Deep Water $\quad-80 \pm 10 \quad-5.0-117 \pm 10$

Coll. May 8, $1957 ; 22^{\circ} 40^{\prime} \mathrm{S}$ Lat, $03^{\circ} 16^{\prime} \mathrm{E}$

Long; $1646 \mathrm{~m}$ depth.

L-410Z. (98) Antarctic Deep Water $-71 \pm 10 \quad-1.0-116 \pm 10$

Coll. April 2, $1957 ; 35^{\circ} 20^{\prime} \mathrm{S}$ Lat, $10^{\circ} 42^{\prime} \mathrm{E}$

Long; $2195 \mathrm{~m}$ depth.

L-410T. (99) Antarctic Deep Water $\quad-100 \pm 5 \quad-6.0-134 \pm 5$

Coll. April 7, $1957 ; 38^{\circ} 58^{\prime} \mathrm{S}$ Lat, $40^{\circ} 06^{\prime}$

W Long; $1829 \mathrm{~m}$ depth.

5) Subsurface samples 2500 to $4000 \mathrm{~m}$

\section{L-287N. (100) North Atlantic Deep Water}

Coll. July 27, $1955 ; 34^{\circ} 55^{\prime} \mathrm{N}$ Lat, $57^{\circ} 11^{\prime}$

$$
-43 \pm 10(-3.7)(-84 \pm 10)
$$

W Long; $3488 \mathrm{~m}$ depth.

L-334X.(101) North Atlantic Deep Water

Coll. April 18, $1956 ; 28^{\circ} 4 \mathrm{I}^{\prime} \mathrm{N}$ Lat, $60^{\circ} 19^{\prime}$

$$
-62 \pm 6 \quad-0.8-107 \pm 6
$$

W Long; $2560 \mathrm{~m}$ depth.

L-367Z. (102) North Atlantic Deep Water

Coll. September 9, $1956 ; 26^{\circ} 10^{\prime} \mathrm{N}$ Lat, $18^{\circ}$

$-62 \pm 8 \quad-1.1-107 \pm 8$

06' W Long; $3503 \mathrm{~m}$ depth.

L-334S. (103) North Atlantic Deep Water $-56 \pm 7 \quad-0.8-102 \pm 7$

Coll. April 16, 1956; $25^{\circ} 01^{\prime} \mathrm{N}$ Lat, $59^{\circ} 11^{\prime}$

W Long; $2560 \mathrm{~m}$ depth.

\section{L-282R. (104) North Atlantic Deep Water}

Coll. June 22, $1955 ; 24^{\circ} 26^{\prime} \mathrm{N}$ Lat, $70^{\circ} 23^{\prime}$

$$
-50 \pm 7(-3.7)(-90 \pm 9)
$$

W Long; $2787 \mathrm{~m}$ depth. 
L-464G. (105) North Atlantic Deep Water $\delta \mathrm{C}^{14} \quad \delta \mathrm{C}^{13} \quad \Delta$ Coll. November 21,$1957 ; 22^{\circ} 03^{\prime} \mathrm{N}$ Lat, $51^{\circ}$

$-53 \pm 6 \quad-7.0-87 \pm 6$ $27^{\prime}$ W Long; $2700 \mathrm{~m}$ depth.

L-464I. (106) North Atlantic Deep Water $-62 \pm 6 \quad-3.0-103 \pm 6$ Coll. November 22,$1957 ; 20^{\circ} 43^{\prime} \mathrm{N}$ Lat, $49^{\circ}$ $26^{\prime}$ W Long; $2926 \mathrm{~m}$ depth.

L-464L. (107) North Atlantic Deep Water $\mathbf{- 6 9} \pm \mathbf{7} \quad \mathbf{- 5 . 6 - 1 0 5} \pm \mathbf{7}$ Coll. November 25,$1957 ; 16^{\circ} 44^{\prime} \mathrm{N}$ Lat, $42^{\circ}$

$38^{\prime}$ W Long; $3840 \mathrm{~m}$ depth.

L-410A. (108) North Atlantic Deep Water $-53 \pm 5 \quad-11.3 \quad-79 \pm 5$ Coll. December 31,$1956 ; 15^{\circ} 52^{\prime} \mathrm{N}$ Lat, $38^{\circ}$

03' W Long; $3840 \mathrm{~m}$ depth.

\section{L-334N. (109) North Atlantic Deep Water $-74 \pm 7 \quad 1.4-123 \pm 7$} Coll. April 11, 1956; $15^{\circ} 49^{\prime} \mathrm{N}$ Lat, $47^{\circ} 12^{\prime}$ W Long; $2560 \mathrm{~m}$ depth.

L-334F. (110) North Atlantic Deep Water $-49 \pm 6 \quad-2.7 \quad-91 \pm 6$ Coll. February 18,$1955 ; 03^{\circ} 48^{\prime} \mathrm{S}$ Lat, $30^{\circ}$

53' W Long; $2560 \mathrm{~m}$ depth.

L-419E. (111) North Atlantic Deep Water $\mathbf{- 6 2} \pm \mathbf{7} \quad \mathbf{- 3 . 0 - 1 0 3 \pm 7}$ Coll. May 27, 1957; $04^{\circ} 23^{\prime} \mathrm{S}$ Lat, $00^{\circ} 15^{\prime}$

W Long; $2743 \mathrm{~m}$ depth.

L-410J. (112) North Atlantic Deep Water $-71 \pm 6 \quad-10.0 \quad-99 \pm 6$ Coll. January 22,$1957 ; 17^{\circ} 03^{\prime} \mathrm{S}$ Lat, $28^{\circ}$

13' W Long; $2807 \mathrm{~m}$ depth.

L-410FF. (113) North Atlantic Deep Water

Coll. May 8, 1957; $22^{\circ} 56^{\prime} \mathrm{S}$ Lat, $04^{\circ} 52^{\prime} \mathrm{E} \quad \begin{array}{ccc}\mathbf{- 6 3} \pm \mathbf{7} & \mathbf{- 5 . 5} & \mathbf{- 9 8} \pm \mathbf{7}\end{array}$ Long; $2560 \mathrm{~m}$ depth.

L-465H. (114) North Atlantic Deep Water $-57 \pm 6 \quad-4.0 \quad-96 \pm 6$ Coll. January 18,$1958 ; 38^{\circ} 25^{\prime} \mathrm{S}$ Lat, $53^{\circ}$

$11^{\prime}$ W Long; $2770 \mathrm{~m}$ depth.

\section{L-287A. (115) North Atlantic Deep Water}

6) Subsurface samples greater than $4000 \mathrm{~m}$

Coll. July 16, $1955 ; 39^{\circ} 27^{\prime}$ N Lat, $56^{\circ} 57^{\prime}-\mathbf{4 3} \pm \mathbf{7}(\mathbf{- 3 . 7})(\mathbf{- 8 4} \pm 9)$

W Long; 4345 m depth.

L-287C. (116) North Atlantic Deep Water

Coll. July 18,$1955 ; 39^{\circ} 03^{\prime} \mathrm{N}$ Lat, $53^{\circ} 29^{\prime}-\mathbf{- 5 8} \pm \mathbf{7}(\mathbf{- 3 . 7})(\mathbf{- 9 8} \pm \mathbf{9})$ W Long; $5281 \mathrm{~m}$ depth. 
$\delta \mathrm{C}^{14} \quad \delta \mathrm{C}^{13} \quad \Delta$

L-287H.(117) North Atlantic Deep Water

$$
-40 \pm 13(-3.7)(-81 \pm 13)
$$

Coll. July 19,$1955 ; 38^{\circ} 00^{\prime} \mathrm{N}$ Lat, $51^{\circ} 37^{\prime}$

W Long; $5369 \mathrm{~m}$ depth.

L-287L. (118) North Atlantic Deep Water

$$
-73 \pm 13(-3.7)(-112 \pm 13)
$$

Coll. July 23,$1955 ; 37^{\circ} 23^{\prime} \mathrm{N}$ Lat, $53^{\circ} 22^{\prime}$

W Long; $5368 \mathrm{~m}$ depth.

L-287M. (119) North Atlantic Deep Water

Coll. July 26,$1955 ; 35^{\circ} 28^{\prime} \mathrm{N}$ Lat, $55^{\circ} 48^{\prime}$

$$
-53 \pm 7(-3.7)(-93 \pm 9)
$$

W Long; $5416 \mathrm{~m}$ depth.

L-287K.(120) North Atlantic Deep Water

Coll. July 22, $1955 ; 35^{\circ} 43^{\prime} \mathrm{N}$ Lat, $53^{\circ} 15^{\prime}$

$$
-58 \pm 5(-3.7)(-98 \pm 9)
$$

W Long; 5454 m depth.

\section{L-287I. (121) North Atlantic Deep Water}

$-76 \pm 13(-3.7)(-115 \pm 13)$

Coll. July 21,$1955 ; 34^{\circ} 46^{\prime} \mathrm{N}$ Lat, $52^{\circ} 46^{\prime}$

W Long; $5481 \mathrm{~m}$ depth.

\section{L-282Y. (122) North Atlantic Deep Water}

Coll. June 27, 1955; $31^{\circ} 47^{\prime} \mathrm{N}$ Lat, $71^{\circ} 13^{\prime}$

$$
-60 \pm 13(-3.7)(-100 \pm 13)
$$

W Long; $5360 \mathrm{~m}$ depth.

\section{L-282Z. (123) North Atlantic Deep Water}

Coll. June 29,$1955 ; 31^{\circ} 21^{\prime} \mathrm{N}$ Lat, $66^{\circ} 39^{\prime}$

$-56 \pm 7(-3.7)(-96 \pm 9)$

W Long; 4893 m depth.

\section{L-282X.(124) North Atlantic Deep Water}

Coll. June 27,$1955 ; 31^{\circ} 17^{\prime} \mathrm{N}$ Lat, $71^{\circ} 03^{\prime}$

$$
-52 \pm 9(-3.7)(-92 \pm 10)
$$

W Long; $5367 \mathrm{~m}$ depth.

\section{L-282W. (125) North Atlantic Deep Water}

Coll. June 26, 1955; $29^{\circ} 14^{\prime} \mathrm{N}$ Lat, $69^{\circ} 55^{\prime}$

$$
-33 \pm 13(-3.7)(-74 \pm 13)
$$

W Long; $5400 \mathrm{~m}$ depth.

\section{L-282L. (127) North Atlantic Deep Water}

Coll. June 15,$1955 ; 25^{\circ} 07^{\prime} \mathrm{N}$ Lat, $69^{\circ} 57^{\prime}$

$$
-53 \pm 20(-3.7)(-93 \pm 20)
$$

W Long; $5508 \mathrm{~m}$ depth. 
$\begin{array}{lllc} & \delta C^{14} & \delta C^{13} & \Delta \\ \text { L-334T. (128) North Atlantic Deep Water } & -88 \pm 6 & -9.0-117 \pm 6\end{array}$

Coll. April 16, $1956 ; 25^{\circ} 01^{\prime} \mathrm{N}$ Lat, $59^{\circ} 12^{\prime}$

W Long; $6035 \mathrm{~m}$ depth.

\section{L-367DD. (129) North Atlantic Deep Water}

Coll. October 11, 1956; $24^{\circ} 23^{\prime} \mathrm{N}$ Lat, $24^{\circ}$

$$
-82 \pm 5-0.2-128 \pm 5
$$

$03^{\prime}$ W Long; $4921 \mathrm{~m}$ depth.

\section{L-282P.(130) North Atlantic Deep Water}

Coll. June 20,$1955 ; 24^{\circ} 05^{\prime} \mathrm{N}$ Lat, $68^{\circ} 23^{\prime}-\mathbf{- 5 8} \pm \mathbf{8}(\mathbf{- 3 . 7})(\mathbf{- 9 8} \pm \mathbf{8})$ W Long; 5584 m depth.

\section{L-282N. (131) North Atlantic Deep Water}

Coll. June 17, 1955; $23^{\circ} 28^{\prime} \mathrm{N}$ Lat, $65^{\circ} 56^{\prime}$

$$
-55 \pm 13(-3.7)(-95 \pm 13)
$$

W Long; $5788 \mathrm{~m}$ depth.

Lr334P. (132) North Atlantic Deep Water $-87 \pm 6 \quad-8.3-118 \pm 6$ Coll. April 14, 1956; $22^{\circ} 22^{\prime}$ N Lat, $54^{\circ} 19^{\prime}$

W Long; $5698 \mathrm{~m}$ depth.

L-410B. (133) North Atlantic Deep Water $-65 \pm 7 \quad 0.6-113 \pm 7$ Coll. December 31, 1956; $15^{\circ} 52^{\prime}$ N Lat, $38^{\circ}$

08' W Long; $5264 \mathrm{~m}$ depth.

L-464N. (134) North Atlantic Deep Water $-83 \pm 9 \quad-5.7-118 \pm 9$ Coll. November 26,$1957 ; 15^{\circ} 29^{\prime} \mathrm{N}$ Lat, $40^{\circ}$

$30^{\circ} \mathrm{W}$ Long; $4147 \mathrm{~m}$ depth.

L-334D. (135) Antaretic Bottom Water $-99 \pm 7 \quad-1.1-142 \pm 7$ Coll. February 16, 1956; $03^{\circ} 26^{\prime} \mathrm{S}$ Lat, $31^{\circ}$

34' W Long; 4389 m depth.

L-410H. (136) Antaretic Bottom Water $-105 \pm 6 \quad-4.2-142 \pm 6$ Coll. January 22, $1957 ; 16^{\circ} 34^{\prime} \mathrm{S}$ Lat, $28^{\circ}$

03' W Long; $5330 \mathrm{~m}$ depth.

\section{L-410N. (137) Antarctic Bottom Water $\quad \mathbf{- 8 8} \pm 5 \quad \mathbf{- 5 . 0}-124 \pm 5$}

Coll. February 1, 1957; $33^{\circ} 43^{\prime} \mathrm{S}$ Lat, $45^{\circ}$

18' W Long; 4380 m depth.

B. $\mathrm{CO}_{2}$ obtained from acidified samples of sea water from Pacific Ocean. Samples in this group coll. by Univ. of Washington research vessel Brown Bear, under the direction of S. El Wardani.

\section{L-470A. North Pacific Basin \\ $-180 \pm 7 \quad 0.3-222 \pm 7$}

Coll. August 15, 1957; $50^{\circ} 55^{\prime} \mathrm{N}$ Lat, $177^{\circ}$

23' E Long; $5200 \mathrm{~m}$ depth. 


\section{$\delta \mathrm{C}^{14} \quad \delta \mathrm{C}^{13} \quad \Delta$ \\ L-470B. Aluetian Trench \\ $-158 \pm 8 \quad-0.9-199 \pm 8$}

Coll. August 17, 1957;51 $01^{\prime} \mathrm{N}$ Lat, $174^{\circ}$

$4 \mathrm{l}^{\prime}$ E Long; $6200 \mathrm{~m}$ depth.

\section{L-470C. North Pacific Surface Water \\ Coll. August 14, $1957 ; 50^{\circ} 42^{\prime} \mathrm{N}$ Lat, $177^{\circ}$}

$$
-34 \pm 5 \quad 0.6 \quad-83 \pm 5
$$

$17^{\prime}$ E Long; 1 m depth.

\section{L-470D. North Pacific Surface Water}

$$
89 \pm 6 \quad 0.9 \quad 33 \pm 6
$$

Coll. August 30,$1957 ; 53^{\circ} 10^{\prime} \mathrm{N}$ Lat, $162^{\circ}$

$22^{\prime}$ W Long; 1 m depth.

C. $\mathrm{CO}_{2}$ obtained from acidified samples of sea water from deep basins of the Caribbean Sea. Samples in this group coll. by the scientific staff of Columbia University research vessel Vema under the direction of M. Ewing, R. Gerard, and B. Heezen. These results are discussed in a paper by Broecker, Gerard, Heezen, and Ewing (in press). The numbers in parentheses beside each laboratory number are the index numbers used by Broecker and others in that paper.

L-529J. ( 138) Columbia Basin $\quad-53 \pm 5 \quad-0.6 \quad-99 \pm 5$

Coll. November 7,$1958 ; 14^{\circ} 05^{\prime} \mathrm{N}$ Lat, $75^{\circ}$

25' W Long; 2316 m depth.

\section{L-419P.(139) Columbia Basin $\quad-49 \pm 7 \quad-5.6 \quad-86 \pm 7$}

Coll. July 29,$1957 ; 17^{\circ} 00^{\prime} \mathrm{N}$ Lat, $74^{\circ} 24^{\prime}$

W Long; 234l m depth.

\section{L-529K. (140) Columbia Basin $\quad-43 \pm 13 \quad-0.8 \quad-89 \pm 13$}

Coll. November 7,$1958 ; 14^{\circ} 05^{\prime} \mathrm{N}$ Lat, $75^{\circ}$

$25^{\prime}$ W Long; $3262 \mathrm{~m}$ depth.
L-529L. (141) Columbia Basin $\quad-49 \pm 7 \quad 0.5 \quad-98 \pm 7$
Coll. November 7,$1958 ; 14^{\circ} 05^{\prime} \mathrm{N}$ Lat, $75^{\circ}$

$25^{\prime}$ W Long; $3790 \mathrm{~m}$ depth.

\section{L-326W. (142) Columbia Basin $\quad-55 \pm 7 \quad-1.0-100 \pm 7$}

Coll. January 1,$1956 ; 17^{\circ} 31^{\prime} \mathrm{N}$ Lat, $73^{\circ}$

$22^{\prime}$ W Long; 4265 m depth.

\section{L-529H. (143) Cayman Trough
Coll. November 5, 1958; $19^{\circ} 31^{\prime} \mathrm{N}$ Lat, $74^{\circ}$}

$59^{\prime}$ W Long; $1647 \mathrm{~m}$ depth.

\section{L-529G. (144) Cayman Trough \\ Coll. November 5,$1958 ; 19^{\circ} 31^{\prime} \mathrm{N}$ Lat, $74^{\circ}$}

59' W Long; 1893 m depth.

\section{L-326S. (145) Cayman Trough \\ Coll. December 23, 1955; $19^{\circ} 15^{\prime} \mathrm{N}$ Lat, $79^{\circ}$}

$-32 \pm 5 \quad-0.5 \quad-79 \pm 5$

$30^{\prime}$ W Long; $2560 \mathrm{~m}$ depth.

$-26 \pm 5 \quad-0.5 \quad-74 \pm 5$

$$
-24 \pm 5 \quad 2.7-78 \pm 5
$$




\section{L-326T. (146) Cayman Trough \\ $\delta \mathrm{C}^{14} \quad \delta \mathrm{C}^{13} \quad \Delta$ \\ Coll. December 24, 1955; $19^{\circ} 10^{\prime} \mathrm{N}$ Lat, $78^{\circ}$

$$
-43 \pm 7 \quad-3.7 \quad-84 \pm 7
$$ \\ $00^{\prime}$ W Long; 4755 m depth.}

\section{L-419Q. (147) Cayman Trough}

Coll. July 30,$1957 ; 19^{\circ} 33^{\prime} \mathrm{N}$ Lat, $75^{\circ} 02^{\prime}$

$$
-28 \pm 7 \quad-5.9-65 \pm 7
$$

W Long; 4844 m depth.

\section{L-419K. (148) Granada Basin}

$-55 \pm 7 \quad-11.3 \quad-81 \pm 7$

Coll. July 11, 1957; $12^{\circ} 45^{\prime} \mathrm{N}$ Lat, $63^{\circ} 06^{\prime}$

W Long; $2468 \mathrm{~m}$ depth.

\section{L-419L. (149) Cariaco Trench}

Coll. July 13,$1957 ; 10^{\circ} 36^{\prime} \mathrm{N}$ Lat, $65^{\circ} 04^{\prime}$

$$
-23 \pm 7 \quad-16.6-39 \pm 7
$$

W Long; $550 \mathrm{~m}$ depth.

\section{L-419M. (150) Cariaco Trench}

Coll. July 14, 1957; $10^{\circ} 39^{\prime} \mathrm{N}$ Lat, $65^{\circ} 43^{\prime}$

$$
3 \pm 7 \quad-7.7-32 \pm 7
$$

W Long; 1152 m depth.

D. Organisms deriving their organic and inorganic carbon from the dissolved $\mathrm{CO}_{2}$ in sea water.

\section{L-444. (38) Benguela Current $\quad-64 \pm 15 \quad-17.7 \quad-78 \pm 15$}

Organic material from plankton coll. at a depth of $73 \mathrm{~m}$ by the scientific staff of Columbia Univ. research vessel $V e m a$ on May 5, $1957\left(22^{\circ}\right.$ $34^{\prime} \mathrm{S}$ Lat, $14^{\circ} 12^{\prime}$ E Long; subm. by R. Menzies, Univ. of Southern California.

\section{L-570. MeMurdo Sound, Antarctica $\quad-148 \pm 7 \quad-24.7-149 \pm 7$}

Flesh from right rear flipper of freshly killed seal ( $77^{\circ} \mathrm{S}$ Lat, $165^{\circ} \mathrm{E}$ Long). Coll. by J. Mulligan, U.S.A.R.P., December 17, 1959; subm. by T. Pewe, U. S. Geol. Survey. Result is in the same range as the $\Delta$ values obtained by Rafter and Fergusson (1958) for the dissolved $\mathrm{CO}_{2}$ in surface waters adjacent to the Antarctic. The low $\mathrm{C}^{14}$ concentration in these waters results in an apparent radiocarbon age of $1300 \mathrm{yr}$ for the marine organisms living in this area.

\section{L-112D. Adak, Aleutian Islands}

Clam shells bearing dried meat from a

$$
-79 \pm 9 \quad 1.3-127 \pm 9
$$
lagoon $\left(51^{\circ} 40^{\prime} \mathrm{N}\right.$ Lat, $176^{\circ} 30^{\prime} \mathrm{W}$ Long). Coll. July 1950 by H. Powers, U. S. Geol. Survey. Whether the low value results from freshwater drainage into the lagoon, the upwelling of deep 
water, or a non-zero age for the shells is not known.

\section{L-584A. Eniwetok Atoll}

Living coral from a reef off Bogan Island ( $11^{\circ} 30^{\prime} \mathrm{N}$ Lat, $162^{\circ} 10^{\prime} \mathrm{E}$ Long). Coll. 1946 by J. Tracey, U. S. Geol. Survey.

\section{L-595. Vancouver Island, B. C.}

Living barnacles $\left(49^{\circ} \mathrm{N}\right.$ Lat, $124^{\circ} \mathrm{W}$ Long $)$. Coll. 1959; subm. by J. Fyles, Geol. Survey of Canada.

\section{L-599A. Bay of Arcachon, France}

Pelecypods (Cardium edule L.) from Atlantic Ocean along the coast of Southwestern France $\left(44^{\circ} 35^{\prime} \mathrm{N}\right.$ Lat, $01^{\circ} 25^{\prime} \mathrm{W}$ Long). Coll. 1952; subm. by W. Zagwijn, Geol. Survey of the Netherlands.

\section{L-599B. Wijk aan Zee, Netherlands}

Pelecypods (Cardium edule L.) from the North Sea coast ( $52^{\circ} 30^{\prime} \mathrm{N}$ Lat, $04^{\circ} 25^{\prime} \mathrm{E}$ Long). Coll. May, 1960 by W. Zagwijn.

\section{L-576A. Jamaica, B.W.I.}

Gastropods (Liiona pica L) coll. 1929 or 1930 ( $18^{\circ} \mathrm{N}$ Lat, $78^{\circ} \mathrm{W}$ Long) ; subm. by $\mathrm{H}$. Rehder, National Museum, Washington, D. C.

\section{L-576F. Jamaica, B.W.I.}

Gastropods (Liiona pica L) coll. 1884. (18º $\mathrm{N}$ Lat, $78^{\circ} \mathrm{W}$ Long); subm. by H. Rehder.

\section{L-576B. Bahama Islands}

Gastropods (Strombus raninus) coll. 1950 ( $26^{\circ} \mathrm{N}$ Lat, $78^{\circ} \mathrm{W}$ Long); subm. by N. Newell, American Museum of Natural History.

\section{L-576G. Bahama Islands}

Gastropods (Strombus raninus) coll. during the 1880s ( $26^{\circ} \mathrm{N}$ Lat, $78^{\circ} \mathrm{W}$ Long); subm. by H. Rehder.

\section{L-593C. Bahama Islands}

Pelecypods (Codakia orbicularis) coll. June 1958 at Bimini Cay $\left(25^{\circ} 45^{\prime} \mathrm{N}\right.$ Lat, $79^{\circ} 15^{\prime} \mathrm{W}$ Long); subm. by J. Imbrie and A. McIntyre, Columbia Univ. $\delta \mathrm{C}^{14} \quad \delta \mathrm{C}^{13} \quad \Delta$

$-30 \pm 5 \quad-2.4 \quad-74 \pm 5$

$-5 \pm 5 \quad-0.3 \quad-54 \pm 5$

$-8 \pm 5(0.3)(-58 \pm 5)$

$-9 \pm 7 \quad(0.3)(-59 \pm 7)$

$$
5 \pm 5 \quad 1.3-48 \pm 5
$$

$10 \pm 5 \quad 1.3-43 \pm 5$

$$
2 \pm 5 \quad 1.4 \quad-51 \pm 5
$$

$-2 \pm 7 \quad 1.6 \quad-55 \pm 7$

$27 \pm 7(0.3)(-25 \pm 7)$ 


\section{L-576C. Kollaf jord, Iceland}

Gastropods (Nucella lapillus L) coll. 1946

$$
\begin{array}{rcc}
\delta \mathrm{C}^{14} & \delta \mathrm{C}^{13} & \Delta \\
-8 \pm 6 & (3.4) & (-64 \pm 6)
\end{array}
$$

from Faxa Bay $\left(22^{\circ} \mathrm{N}\right.$ Lat, $64^{\circ} \mathrm{W}$ Long); subm. by H. Einarsson, Univ. Research Institute, Reykjavik, Iceland.

\section{L-576H. Kollaf jord, Iceland}

Gastropods (Nucella lapillus L) coll. 1900

$$
-13 \pm 6 \quad 3.4 \quad-69 \pm 6
$$

from Faxa Bay $\left(22^{\circ} \mathrm{N}\right.$ Lat, $64^{\circ} \mathrm{W}$ Long) ; subm. by G. Thorson, Univ. of Copenhagen.

\section{L-576I. Kollaf jord, Iceland}

Gastropods (Nucella lapillus L) coll. 1840

$$
-16 \pm 6 \quad 3.4 \quad-72 \pm 6
$$

from Faxa Bay $\left(22^{\circ} \mathrm{N}\right.$ Lat, $64^{\circ} \mathrm{W}$ Long $)$; subm. by G. Thorson.

\section{L-576J. Oahu, Hawaii}

Gastropods (Trechus intertextus) coll. 1840

$$
-8 \pm 6 \quad 2.2-62 \pm 6
$$

or 1841 $\left(22^{\circ} \mathrm{N}\right.$ Lat, $158^{\circ} \mathrm{W}$ Long); subm. by

H. Rehder.

\section{L-576D. Pearl Harbor, Hawaii}

Gastropods (Trechus intertextus) coll. 1936 $\left(22^{\circ} \mathrm{N}\right.$ Lat, $158^{\circ} \mathrm{W}$ Long) ; subm. by H. Rehder.

\section{L-576E. Tahiti}

Gastropods (Turbo setosus) coll. 1957 (18

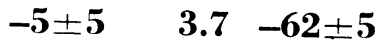

S Lat, $149^{\circ} \mathrm{W}$ Long) ; subm. by H. Rehder.

\section{L-576K. Moorea}

Gastropods (Turbo setosus) coll. early 1880s

$$
\begin{array}{llll}
2 \pm 5 & 4.7 & -58 \pm 5
\end{array}
$$

( $18^{\circ} \mathrm{S}$ Lat, $149^{\circ} \mathrm{W}$ Long); subm. by H. Rehder.

III. SAMPLES UTILIZING $\mathrm{CO}_{2}$ FROM TERRESTRIAL WATERS

\section{A. Lake Samples}

\section{L-487J. Mono Lake, California}

$$
-105 \pm 15
$$

$4.0-157 \pm 15$

Dissolved $\mathrm{CO}_{2}$ coll. by passing lake waters through an anion exchange column $\left(37^{\circ} 59^{\prime} \mathrm{N}\right.$ Lat, $119^{\circ} 08^{\prime} \mathrm{W}$ Long). Coll. August 23, 1958 by O. Schaeffer, Brookhaven National Laboratory.

\section{L-415GGG. Great Salt Lake, Utah}

$$
-93 \pm 6 \quad 2.1-142 \pm 6
$$

Dissolved $\mathrm{CO}_{2}$ coll. by acidifying lake water and bubbling with $\mathrm{N}_{2}$. Coll. $\left(40^{\circ} 50^{\circ} \mathrm{N}\right.$ Lat, $112^{\circ}$ $35^{\prime} \mathrm{W}$ Long) June 1958 by R. Cohenour, Salt Lake City, Utah (Broecker and Olson, 1960). 


\section{L-542. Great Salt Lake, Utah}

Brine shrimp $\left(40^{\circ} 50^{\prime} \mathrm{N}\right.$ Lat, $112^{\circ} 35^{\prime} \mathrm{W}$ Long) ; coll. August 1959 by C. Sanders, Ogden, Utah (Broecker and Olson, 1960).

\section{B. River Samples}

\section{L-487F. San Francisco Flycasting Club}

Pelecypods (Margaritifera margaritifera falcata (Gould) ) living on a sandy shoal in Truckee River ca. 5 mi E of Truckee, California $\left(39^{\circ} 23^{\prime}\right.$ $\mathrm{N}$ Lat, $120^{\circ} 07^{\prime} \mathrm{W}$ Long). Coll. August 25, 1958 by W. Broecker and O. Schaeffer (Broecker and Olson, 1960).

\section{L-487G. San Francisco Flycasting Club}

Meat from the above shells (L-487F) (Broecker and Olson, 1960).

\section{L-487H. San Francisco Flycasting Club}

Dissolved $\mathrm{CO}_{2}$ coll. by passing river water through an anion exchange column. From same locality as L-487F. Coll. August 25, 1958 (Broecker and Olson, 1960).

\section{L-415S. Ogden, Utah}

Plants growing within waters of Weber River near Rt. 91 bridge $\left(41^{\circ} 10^{\prime} \mathrm{N}\right.$ Lat, $111^{\circ} 50^{\prime} \mathrm{W}$ Long). Coll. September 1957 by A. Walton and W. Broecker (Broecker and Olson, 1960).

\section{L-4870. Ogden, Utah}

Plants growing within waters of Weber River near Rt. 91 bridge $\left(41^{\circ} 10^{\prime} \mathrm{N}\right.$ Lat, $111^{\circ} 50^{\prime} \mathrm{W}$ Long). Coll. September 10, 1958 by W. Broecker (Broecker and Olson, 1960).

\section{Ground Water Samples}

\section{L-500C. Onandaga Cave, Missouri - \\ Dripstone carbonate ca. $3 \mathrm{~mm}$ thick, removed}

$$
-8 \pm 6 \quad-4.8-48 \pm 6
$$

$-22 \pm 6 \quad-22.0-28 \pm 6$

$$
-1 \pm 7 \quad-10.2-31 \pm 7
$$

$-146 \pm 6-33.9-131 \pm 6$ from broken rock used to define a pathway through the cave $\left(38^{\circ} 02^{\prime} \mathrm{N}\right.$ Lat, $97^{\circ} 15^{\prime} \mathrm{W}$ Long). Coll. November 8, 1958 by W. Broecker and E. Olson.

\footnotetext{
L-451C. Crystal Palace Cave, California $\quad-63 \pm 8 \quad-8.9 \quad-93 \pm 8$

Dripstone carbonate, coating and replacing a piece of lumber brought into the cave by miners
} 
ca. 1880. The recency of the wood was confirmed

by a radiocarbon analysis $(\Delta=-5 \pm 10$.) Subm. by R. de Saussure, Berkeley, California.

\section{Hot Springs Sample}

\section{L-487P. Bridgeport, California}

Carbonate forming a one-quarter-inch coating on a wooden bathtub fed by a hot spring $\left(38^{\circ}\right.$ $08^{\prime} \mathrm{N}$ Lat, $119^{\circ} 10^{\prime} \mathrm{W}$ Long). Despite exposure to the atmosphere, sample contained only $2.0 \pm$ $0.8 \%$ of the activity of the oxalic-acid standard. Coll. September 1958 by P. Orr and W. Broecker.

\section{REFERENCES}

Bowen, V. T., and Sugihara, L. T., 1960, Strontium-90 in the "mixed layer" of the Atlantic Ocean: Nature, v. 186, p. $71-72$.

Broecker, W. S., Gerard, R., Ewing, M., and Heezen, B. C., 1960, Natural radiocarbon in the Atlantic Ocean: Jour. Geophys. Research, v. 55, p. 2903-2931.

Broecker, W. S., Gerard, R. S., Heezen, B. C., and Ewing, M., in press, Radiocarbon in the Caribbean Sea: Jour. Geophys. Research.

Broecker, W. S., and Olson, E. A., 1959, Lamont radiocarbon measurements VI: Am. Jour. Sci. Radioc. Supp., v. 1, p. 111-132.

Broecker, W. S, Oldiocarbon from nuclear tests II: Science, v. 132, p. 712-721. Science, v. 7 , p. 331-332., and Schulert, A., 1959, Bomb carbon-14 in human beings:
.

Broecker, W. S., Tucek, C. S., and Olson, E. A., 1959, Radio-carbon analysis of ocean $\mathrm{CO}_{2}$ : Internat. Jour. Applied Radiation and Isotopes, v. 7, p. 1-18. Craig, Harmon, 1951, Mass-spectrometer analyses of radiocarbon standards: Radiocarbon,
v. 3, p. 1-3.

Fonselius, Stig, and Östlund, G., 1959, Natural radiocarbon measurements on surface water from the North Atlantic and Arctic Sea: Tellus, v. 11, p. 77-82.
fter, T. A., and Fergusson G. J

Rafter, T. A., and Fergusson, G. J., 1958, Atmospheric radiocarbon as a tracer in geophysical circulation problems: 2nd United Nations Internat. Conf. on Peaceful Uses of Atomic Energy, v. 18, p. 526-532. 